\title{
On uniformly fully inert subgroups of abelian groups
}

https://doi.org/10.1515/taa-2020-0002

Received March 23, 2019; accepted January 31, 2020

\begin{abstract}
If $H$ is a subgroup of an abelian group $G$ and $\phi \in \operatorname{End}(G), H$ is called $\phi$-inert (and $\phi$ is $H$-inertial) if $\phi(H) \cap H$ has finite index in the image $\phi(H)$. The notion of $\phi$-inert subgroup arose and was investigated in a relevant way in the study of the so called intrinsic entropy of an endomorphism $\phi$, while inertial endomorphisms (these are endomorphisms that are $H$-inertial for every subgroup $H$ ) were intensively studied by Rinauro and the first named author.

A subgroup $H$ of an abelian group $G$ is said to be fully inert if it is $\phi$-inert for every $\phi \in \operatorname{End}(G)$. This property, inspired by the "dual" notion of inertial endomorphism, has been deeply investigated for many different types of groups $G$. It has been proved that in some cases all fully inert subgroups of an abelian group $G$ are commensurable with a fully invariant subgroup of $G$ (e.g., when $G$ is free or a direct sum of cyclic $p$-groups). One can strengthen the notion of fully inert subgroup by defining $H$ to be uniformly fully inert if there exists a positive integer $n$ such that $|(H+\phi H) / H| \leq n$ for every $\phi \in \operatorname{End}(G)$. The aim of this paper is to study the uniformly fully inert subgroups of abelian groups. A natural question arising in this investigation is whether such a subgroup is commensurable with a fully invariant subgroup. This paper provides a positive answer to this question for groups belonging to several classes of abelian groups.
\end{abstract}

Keywords: fully invariant subgroup, $\phi$-inert subgroup, fully inert subgroup, uniformly fully inert subgroup, commensurable subgroup

2010 MSC: Primary: 20K27, 20K30; Secondary: 20K15, 20K21

Dedicated to the memory of Adalberto Orsatti

\section{Introduction}

Unless otherwise stated, all groups considered in this paper are assumed to be abelian. For unexplained notions and notation we refer to the recent monograph by Laszlo Fuchs [21].

\subsection{Levels of invariance in the lattice of subgroups}

If $\phi$ is an endomorphism of a group $G$ and $H$ a subgroup of $G$, then $H$ is said to be $\phi$-invariant if $\phi H \leq H$. A subgroup $F$ of a group $G$ is fully invariant if it is $\phi$-invariant for every endomorphism $\phi$ of $G$. Fully invariant subgroups have been an important tool in the theory of abelian groups; they form a complete sublattice of the lattice $\mathcal{L}(G)$ of all subgroups of $G$, which is denoted by $\operatorname{Jn} v(G)$.

\footnotetext{
^Corresponding Author: Ulderico Dardano: Dipartimento di Matematica e Applicazioni R.Caccioppoli, Università di Napoli Federico II, Via Cintia - Monte S. Angelo, 80126 Napoli, Italy, E-mail: ulderico.dardano@unina.it

Dikran Dikranjan: Dipartimento di Matematica ed Informatica, Università di Udine, Via delle Scienze, 208, 33100 Udine, Italy, E-mail: dikran.dikranjan@uniud.it

Luigi Salce: Dipartimento di Matematica ”Tullio Levi-Civita”, Università di Padova, Via Trieste 63 - 35121 Padova, Italy, E-mail: salce@math.unipd.it
} 
It may happen that the group $G$ has only the two trivial fully invariant subgroups (this occurs precisely when $G$ is a simple module over the ring $\operatorname{End}(G)$ ). This makes it natural to replace the somewhat too restrictive relation of invariance of a subgroup with respect to an endomorphism by a weaker condition which is trivially satisfied also by all finite subgroups and all subgroups of finite index.

Definition 1.1. Let $\phi$ be an endomorphism and $H$ a subgroup of a group $G$. Then $H$ is called $\phi$-inert if $\phi(H) \cap H$ has finite index in $\phi(H)$

In the above situation, one can also say that $\phi$ is $H$-inertial. Note that $H$ is $\phi$-inert precisely when $(H+\phi H) / H$ is finite.

Asking a subgroup $H$ to be $\phi$-inert for all endomorphisms $\phi$, one obtains the following notion, introduced in [17].

Definition 1.2. [17] A subgroup $H$ of a group $G$ is fully inert if $H$ is $\phi$-inert for every endomorphism $\phi$ of $G$.

We recall that the notion of $\phi$-inert subgroup was strongly motivated by the study of the dynamical properties of the endomorphism $\phi$ in [17], while fully inert subgroups provide a generalization of fully invariant subgroups.

Exchanging the quantifier, one can say that an endomorphism $\phi$ of the group $G$ such that $H$ is $\phi$-inert for each subgroup $H$ of $G$ is an inertial endomorphism. Inertial endomorphisms are investigated in [10] and [11].

A short digression to the non-commutative setting is now in order, showing the parallel development of similar notions in the non-commutative and in the abelian setting. It is worthwhile observing that the notion of inertial automorphisms was introduced in [9] and inspired by a well-known notion in the context of noncommutative groups. Namely, a subgroup $H$ of a group $G$ is called inert if the index $\left|H: H^{g} \cap H\right|$ is finite for all $g \in G$ (where $H^{g}=g^{-1} H g$ ). Clearly this is equivalent to $\left|H^{g}: H^{g} \cap H\right|$ being finite for all $g \in G$, since $\left|H^{g}: H \cap H^{g}\right|=\left|H: H^{g-1} \cap H\right|$.

Inert subgroups seem to have been introduced in 1993 in papers of Belyaev as a tool in the investigation of infinite simple groups (see [2]). Clearly normal subgroups and finite subgroups are inert. All subgroups of an abelian group are trivially inert. On the other hand, a subgroup $H$ of a non-commutative group $G$ which is fully inert (as in Definition 1.2) is actually inert.

The following notion plays a crucial role in both the non-commutative and the abelian settings.

Definition 1.3. Two subgroups $H$ and $K$ of a group $G$ are called commensurable (in symbols, $H \sim K$ ) if $H \cap K$ has finite index both in $H$ and in $K$.

In this terminology, a subgroup $H$ is inert if it is commensurable with all its conjugates $H^{g}$ with $g \in G$. Since commensurability is an equivalence relation in the lattice of subgroups, a subgroup $H$ of $G$ which is commensurable with a normal subgroup of $G$ must be inert in Belyaev's terminology (see [8] for details and proofs; see also Proposition 2.1 for a sharper result).

According to [8], a subgroup $H$ of a group $G$ is said to be uniformly inert if there is $n \in \mathbb{N}$ such that $\left|H: H^{g} \cap H\right| \leq n$ for each $g \in G$. In these terms, Bergman and Lenstra proved the following

Theorem 1.4. [3, Theorem 3] A subgroup of a group is uniformly inert if and only if it is commensurable with a normal subgroup of the group.

P. Neumann pointed out that the alternating group on countably many objects has an abelian subgroup which is inert but not uniformly inert (see details in [8]). Moreover, there exist also soluble finitely generated groups with subgroups which are inert but not uniformly inert (see Remark 2.15 below). Groups in which all subgroup are uniformly inert have been studied in [4]. 
Coming back to abelian groups and their endomorphisms, we note that the relevant novelty in the point of view adopted in [17] is the binary relation imposed by Definition 1.1 between a single pair of a subgroup and an endomorphism. This has the advantage of allowing one to extend this binary relation to arbitrary pairs of a family of subgroups and a family of endomorphisms.

\subsection{Uniformly fully inert subgroups}

The possibility of distinguishing "uniformly" inert subgroups was only quite recently pointed out in [8], in accordance with the following definition.

Definition 1.5. A subgroup $H$ of a group $G$ is uniformly fully inert if there exists a positive integer $n$ such that $|\phi H /(H \cap \phi H)| \leq n$ for every endomorphism $\phi$ of $G$. The least common multiple of the numbers $|\phi H /(H \cap \phi H)|$ when $\phi$ ranges over all endomorphisms of $G$ is called the inertial bound of $H$ in $G$ and is denoted by $\mathbf{i b}(H)$.

In analogy to Definition 1.5, one may call $\phi \in \operatorname{End}(G)$ uniformly inertial if there exists a positive integer $n$ such that $|\phi H /(H \cap \phi H)| \leq n$ for every $H \in \mathcal{L}(G)$.

The following conjecture for uniformly fully inert subgroups is naturally suggested by Theorem 1.4.

Conjecture 1.6. [8, Conjecture 5.2] Every uniformly fully inert subgroup of a group $G$ is commensurable with a fully invariant subgroup.

In order to support the conjecture, we recall that in Corollary 1.9 of [8] it is proved that a subgroup $H$ of a group $G$ is commensurable with a characteristic subgroup of $G$ if and only if there is an $n \in \mathbb{N}$ such that $|\gamma H /(H \cap \gamma H)| \leq n$ for each automorphism $\gamma$ of $G$.

The two families of fully inert subgroups and uniformly fully inert subgroups of $G$ are denoted, respectively, by $\mathcal{J}(G)$ and $\mathcal{J}_{u}(G)$. According to [17], a subgroup commensurable with a fully inert subgroup is also fully inert, so, in particular, a subgroup $H$ commensurable with a fully invariant subgroup is fully inert. In $[8$, p. 7$]$ it is stated that such a subgroup $H$ is even uniformly fully inert. For the sake of completeness, in Proposition 2.1 below we give a detailed proof of this fact. If we denote by $\operatorname{In\tilde {v}}(G)$ the set of subgroups of $G$ which are commensurable with fully invariant subgroups, then the following is a chain of sublattices of the whole lattice $\mathcal{L}(G)$ of subgroups of $G$ (see [8, Proposition 1.11] and the text in front of the proposition):

$$
\operatorname{Jn} v(G) \subseteq \mathcal{J} n \tilde{v}(G) \subseteq \mathcal{J}_{u}(G) \subseteq \mathcal{J}(G) \subseteq \mathcal{L}(G) .
$$

Conjecture 1.6 amounts to state that the following equality holds:

$$
\operatorname{Jn} \tilde{v^{2}}(G)=\mathcal{J}_{u}(G) .
$$

Let us mention that the above chain ( $\left.{ }^{\star}\right)$ may collapse for some groups $G$, i.e., $\operatorname{Jn} v(G)=\mathcal{L}(G)$. This occurs, for example, for those groups $G$ such that $\operatorname{End}(G) \cong \mathbb{Z}$; these groups are necessarily torsion-free, and it is known that they can have arbitrarily large cardinality (see [21, Chapter 12, Theorem 4.9]). A torsion example is furnished by the Prüfer group $\mathbb{Z}\left(p^{\infty}\right)$, whose endomorphism ring is $\mathbb{J}_{p}$, the ring of the $p$-adic integers.

All notions introduced above can be extended verbatim from the category $\operatorname{Mod}(\mathbb{Z})$ of abelian groups to the category $\operatorname{Mod}\left(\mathbb{J}_{p}\right)$ of $\mathbb{J}_{p}$-modules. So many questions regarding the above chain ${ }^{\star}$ ) can be formulated for $\mathbb{J}_{p}$-modules, Conjecture 1.6 included.

The study of the interrelations between the lattices in the chain $\left({ }^{\star}\right)$ takes a big portion of [8, Section 5], and several papers have been devoted recently to investigate, in particular, when the equality $\operatorname{In} \tilde{v}(G)=\mathcal{J}(G)$, or the strict inclusion $\operatorname{Inv} \tilde{v}(G) \subsetneq \mathcal{J}(G)$, hold.

On the one hand, examples of fully inert subgroups which fail to be commensurable with fully invariant subgroups of a given group $G$ cannot be found in case $G$ is a free group, or a direct sum of cyclic $p$-groups, or, 
passing to $\mathbb{J}_{p}$-modules, in case of a torsion-free $\mathbb{J}_{p}$-module which is either free or complete. In other words, the equality $\operatorname{Jn} \tilde{v^{\sim}}(G)=\mathcal{J}(G)$ holds for these groups. Thus, a fortiori, the equality $\mathcal{J} n \tilde{v^{\prime}}(G)=\mathcal{J}_{u}(G)$ holds as well. These facts are the main results in the three papers [19], [24] and [25], respectively.

On the other hand, an example of a separable $p$-group $G$ which admits a subgroup $H \in \mathcal{J}(G) \backslash \operatorname{In} v \sim(G)$ is provided in [24]. Furthermore, an example of a torsion-free $\mathbb{J}_{p}$-module $A$ admitting a submodule $K \in \mathcal{J}(A) \backslash$ Inv $\sim(A)$ is provided in [25]. A careful analysis of the above examples shows that the subgroup $H$ and the submodule $K$ are not even uniformly fully inert (see Section 6). Therefore we have the more precise strict inclusions $\mathcal{J}_{u}(G) \subsetneq \mathcal{J}(G)$ and $\mathcal{J}_{u}(A) \subsetneq \mathcal{J}(A)$. Thus, given an arbitrary abelian group $G$ and an arbitrary $\mathbb{J}_{p^{-}}$ module $A$, it is natural to ask what can be said about the inclusions

$$
\operatorname{Jn} \tilde{v^{2}}(G) \subseteq \mathcal{J}_{u}(G) \text { and } \operatorname{Jn} \tilde{v^{2}}(A) \subseteq \mathcal{J}_{u}(A) .
$$

No examples are up to now available ensuring the strict inclusions, thus providing further support to Conjecture 1.6.

The strict inclusion $\mathfrak{I n \sim} \sim(G) \subsetneq \mathcal{J}(G)$ is provided also by divisible groups $G$ whose torsion-free $\operatorname{rank} r k_{0}(G)$ is finite and non-zero. Moreover, $\operatorname{Inv} \tilde{v}(G) \subsetneq \mathcal{J}(G)$ is proved by a recent result by Chekhlov [6], who characterized the completely decomposable groups $G$ of finite rank satisfying this strict inclusion (see $\S 3.2$ for more details).

The main goal of this paper is to study the uniformly fully inert subgroups of abelian groups and to present old and new results related to Conjecture 1.6.

In Section 2 we present some preliminary basic results and introduce the fully invariant hull and the fully invariant core of a subgroup, which are useful tools in our investigation.

In Section 3 we consider the chain $\left({ }^{\star}\right)$ for divisible groups and completely decomposable groups recalling some known facts from [8] and [6, 7], respectively.

In Section 4 we introduce three classes of groups which are of interest for our conjecture. The first one is the class of Orsatti groups, which are the groups compact in the natural topology and whose structure was determined by Orsatti in [28]. The Orsatti groups are algebraically compact groups satisfying certain finiteness conditions. The second class consists of groups with finite ranks, i.e., groups of finite torsion-free rank and finite $p$-ranks for all primes $p$. The third larger class contains the Orsatti groups and the groups with finite ranks and consists of the narrow groups $G$, defined by the property that $G / n G$ is finite for all positive integers $n$ (Definition 4.1). These groups have been characterized in several ways in [17, Theorem 3.3], where it was proved that the adjoint algebraic entropy of any endomorphism of a narrow group is zero (see [17, Proposition 3.7]). The narrow groups are exactly the totally bounded groups in the natural topology.

In Section 5 we prove that our Conjecture 1.6 holds for groups with finite ranks (see Proposition 5.4) and for Orsatti groups (see Theorem 5.8).

As mentioned above, in Section 6 we give detailed proofs of the facts that the examples for the strict inclusions $\mathfrak{J} n \tilde{v}(G) \subsetneq \mathcal{J}(G)$, for $G$ a $p$-group, and $\mathfrak{J} n \tilde{v}(A) \subsetneq \mathcal{J}(A)$, for $A$ a $\mathbb{J}_{p}$-module, are not even uniformly fully inert.

Section 7 summarizes the state of art concerned with the conjecture and collects some open questions related to this conjecture.

Finally, we remark that many of the notions that we introduce in this paper, as well as some statements and proofs, can be carried out in the non-abelian context, see Remark 2.15 for more details.

\section{Notation and terminology}

For an abelian group $G$ we denote by $d(G)$ the maximal divisible subgroup, by $t(G)$ the maximal torsion subgroup, by $t_{p}(G)$ the $p$-primary component of $t(G)$. The order of an element $g \in G$ is denoted by $o(g)$. We follow [21, Chapter 3, Section 4] in defining the $\operatorname{rank} r k(G)$ of a group $G$ as the cardinal number of a maximal linearly independent system containing only elements of infinite and prime power orders. The cardinality of the subset of elements of infinite order is denoted by $r k_{0}(G)$ and is called the torsion-free rank, while the cardinality of the subset of elements of order a power of the prime $p$ is denoted by $r k_{p}(G)$ and is called the $p$-rank of $G$. 
It is well known that $r k_{0}(G)=\operatorname{dim}_{\mathbb{Q}}(G \otimes \mathbb{Q})$ and that $r k_{p}(G)$ is the dimension of the socle $G[p]$ of $t_{p}(G)$ as vector space over $\mathbb{Z} / p \mathbb{Z}$. We will say that $G$ is a group with finite ranks when $r k_{0}(G)<\infty$ and $r k_{p}(G)<\infty$ hold for all primes $p$ (obviously, this does not imply $r k(G)<\infty$ ).

If $G=\bigoplus_{i} G_{i}$ is a direct sum, for each $i$ we will denote by $\pi_{i}$ the projection $\pi_{i}: G \rightarrow G_{i}$.

\section{Preliminary results}

We start this section by proving the fact mentioned in the Introduction, namely that a subgroup commensurable with a fully invariant subgroup is uniformly fully inert; we will freely use the following well-known facts holding for a group $G$ : if $G_{1} \leq G_{2} \leq G$ with $\left|\frac{G_{2}}{G_{1}}\right|<\infty$, then for any $X \leq G$ and any $\phi \in \operatorname{End}(G)$, both $\left|\frac{\phi\left(G_{2}\right)}{\phi\left(G_{1}\right)}\right|$ and $\left|\frac{G_{2} \cap X}{G_{1} \cap X}\right|$ divide $\left|\frac{G_{2}}{G_{1}}\right|$.

Proposition 2.1. Let $H \sim K$ be commensurable subgroups of a group $G$ and $\phi \in \operatorname{End}(G)$.

(a) if $H$ is $\phi$-inert, then $K$ is $\phi$-inert;

(b) if $H$ is fully inert in $G$, then $K$ is fully inert;

(c) if $H$ is uniformly fully inert in $G$, then $K$ is uniformly fully inert, and the inertial bound $\mathbf{i b}(K)$ divides $\mathbf{i b}(H) \cdot|H /(H \cap K)| \cdot|K /(H \cap K)|$.

Proof. Consider the chain

$$
\phi(K) \geq \phi(K) \cap \phi(H) \geq \phi(K) \cap \phi(H) \cap H \geq \phi(H) \cap \phi(K) \cap H \cap K .
$$

We have that the first factor $\frac{\phi(K)}{\phi(K) \cap \phi(H)}$ of (2.1) has order which divides $\left|\frac{K}{H \cap K}\right|$ since it is an epic image of $\frac{\phi(K)}{\phi(K \cap H)}$ which in turn has order dividing $\left|\frac{K}{H \cap K}\right|$.

The second factor group $\frac{\phi(K) \cap \phi(H)}{\phi(K) \cap \phi(H) \cap H}$ of (2.1) has order dividing $\left|\frac{\phi(H)}{H \cap \phi(H)}\right|$ (intersect both numerator and denominator of $\frac{\phi(H)}{\phi(H) \cap H}$ by $\left.\phi(K)\right)$.

Similarly, the third factor $\frac{\phi(K) \cap \phi(H) \cap H}{\phi(H) \cap \phi(K) \cap H \cap K}$ has order dividing $\left|\frac{H}{K \cap H}\right|$ (intersect both numerator and denominator by $\phi(H) \cap \phi(K))$. Thus

$$
\left|\frac{\phi(K)}{\phi(H) \cap \phi(K) \cap H \cap K}\right| \text { divides }\left|\frac{\phi(H)}{\phi(H) \cap H}\right| \cdot\left|\frac{H}{H \cap K}\right| \cdot\left|\frac{K}{H \cap K}\right| \cdot
$$

On the other hand

$$
\phi(K) \geq \phi(K) \cap K \geq \phi(H) \cap \phi(K) \cap H \cap K,
$$

so that

This proves that

$$
\left|\frac{\phi(K)}{\phi(K) \cap K}\right| \text { divides }\left|\frac{\phi(K)}{\phi(H) \cap \phi(K) \cap H \cap K}\right|
$$

$$
\left|\frac{\phi(K)}{\phi(K) \cap K}\right| \text { divides }\left|\frac{\phi(H)}{\phi(H) \cap H}\right| \cdot\left|\frac{H}{H \cap K}\right| \cdot\left|\frac{K}{H \cap K}\right| \cdot
$$

Obviously, (2.2) implies (a) and (c). It only remains to note that (b) follows from (a).

From item (c) of Proposition 2.1 we immediately derive the following

Corollary 2.2. A subgroup commensurable with a fully invariant subgroup of a group $G$ is uniformly fully inert, that is, $\operatorname{Jnv} \tilde{v}(G) \subseteq \mathcal{J}_{u}(G)$. 


\subsection{Uniformly fully inert subgroups of finite direct sums}

Let us see how the study of (uniformly) fully inert subgroups of finite direct sums can be reduced to box-like subgroups. Recall that a subgroup $H$ of an arbitrary direct sum $G=\bigoplus_{i \in I} G_{i}$ is called box-like if it is of the form $H=\bigoplus_{i \in I} H_{i}$ with $H_{i} \leq G_{i}$ for each $i \in I$.

In general, we denote $G_{(i)}=\bigoplus_{j \neq i} G_{j}$ for each $i \in I$. Then, for any $H \leq G, \pi_{i}(H)=\left(H+G_{(i)}\right) \cap G_{i}$ is the image of the canonical projection of $H$ in $G_{i}$. Moreover $H_{i}=H \cap G_{i}=H \cap \pi_{i}(H)$. It follows that any subgroup $H$ is sandwiched between two box-like subgroups:

$$
H_{\diamond}=\bigoplus_{i \in I} H_{i} \leq H \leq \bigoplus_{i \in I} \pi_{i}(H)=H^{\diamond} .
$$

Using this notation, we have the following lemmas. We omit the easy proof of the first one, since it is similar to the proof of Lemma 2.4, which is in turn inspired by [16, Lemma 4.1] concerning fully inert subgroups of divisible groups.

Lemma 2.3. For a subgroup $H$ of a direct sum $G=\bigoplus_{i \in I} G_{i}$ the following are equivalent:

(1) $H$ is a box-like subgroup;

(2) $H_{\diamond}=H$;

(2') $H=H^{\diamond}$;

(3) $H$ is $\pi_{i}$-invariant for all projections $\pi_{i}$.

Lemma 2.4. For a subgroup $H$ of a finite direct sum $G=G_{1} \oplus \cdots \oplus G_{n}$ the following are equivalent:

(1) $H$ is commensurable with some box-like subgroup;

(2) $\left|H^{\diamond} / H_{\diamond}\right|$ is finite;

(3) $H$ is $\pi_{i}$-inert for all projections $\pi_{i}$.

Proof. If (1) holds and $H \sim K=K_{\diamond}$ where $K$ is box-like, then $K$ is $\pi_{i}$-invariant by Lemma 2.3. Then $H$ is $\pi_{i}$-inert by Proposition 2.1 and (3) holds.

Let us assume that (3) holds. We have

$$
H_{i}=H \cap G_{i}=H \cap \pi_{i}(H) \leq \pi_{i}(H)
$$

where $\left|\pi_{i}(H) /\left(H \cap \pi_{i}(H)\right)\right|=\left|\pi_{i}(H) / H_{i}\right|=m_{i}$ is finite. Therefore,

$$
\frac{H^{\diamond}}{H_{\diamond}}=\frac{\bigoplus_{i=1}^{n} \pi_{i}(H)}{\bigoplus_{i=1}^{n} H_{i}} \simeq \bigoplus_{i=1}^{n} \frac{\pi_{i}(H)}{H_{i}}
$$

has order bounded by $m_{1} \cdot \ldots \cdot m_{n}$. So that (2) holds.

Finally (2) implies (1), by ( $\diamond)$.

An immediate consequence is the following

Corollary 2.5. Let $H$ be a subgroup of a finite direct sum $G=G_{1} \oplus \cdots \oplus G_{n}$. If $H$ is uniformly fully inert in $G$ with inertial bound $m$, then $\left|H^{\diamond} / H_{\diamond}\right| \leq m^{n}$ so that both $H^{\diamond}$ and $H_{\diamond}$ are uniformly fully inert and commensurable with $H$.

Proof. Since $H$ satisfies item (3) in Lemma 2.4, arguing as in the proof of that lemma we get that $\left|H^{\diamond} / H_{\diamond}\right| \leq m^{n}$. Now Corollary 2.2 applies.

The next result is inspired by [17, Proposition 4.2].

Proposition 2.6. Let $H=H_{1} \oplus \cdots \oplus H_{n}$ be a box-like subgroup of a group $G=G_{1} \oplus \cdots \oplus G_{n}$. Then $H$ is uniformly fully inert in $G$ if and only if there is a positive integer $r$ such that for each pair of indices $i, j \in\{1, \ldots, n\}$ and every homomorphism $\phi_{i j}: G_{i} \rightarrow G_{j}$ the following inequality holds:

$$
\left|\left(\phi_{i j}\left(H_{i}\right)+H_{j}\right) / H_{j}\right| \leq r .
$$


In particular, under such a condition, for each i the subgroup $H_{i}$ is uniformly fully inert in $G_{i}$ and $\mathbf{i b}\left(H_{i}\right)$ divides $\mathbf{i b}(H)$.

Proof. We may assume $n>1$ and point out that -with some harmless abuse of notation- each $\phi \in \operatorname{End}(G)$ can be written as

$$
\phi=\sum_{i, j=1}^{n} \phi_{i j}
$$

where each $\phi_{i, j}: G_{i} \rightarrow G_{j}$ is a restriction of $\phi$. Then for each $\phi_{i, j} \in \operatorname{Hom}\left(G_{i}, G_{j}\right)$ we have

$$
\frac{\phi_{i j}\left(H_{i}\right)+H_{j}}{H_{j}} \simeq \frac{\left(\phi_{i j}\left(H_{i}\right)+H_{j}\right) \oplus H_{(j)}}{H_{j} \oplus H_{(j)}} \simeq \frac{\phi_{i j}\left(H_{i}\right)+H}{H} \leq \frac{\phi(H)+H}{H}
$$

where $H_{(j)}=\bigoplus_{i \neq j} H_{i}$. Conversely, for each $\phi \in \operatorname{End}(G)$ in the above notation we have

$$
\frac{\phi(H)+H}{H}=\frac{\sum_{i, j} \phi_{i j}(H)+H}{H}=\sum_{i, j=1}^{n} \frac{\phi_{i j}\left(H_{i}\right)+H}{H}
$$

where the last group is an epic image of

$$
\bigoplus_{i, j=1}^{n} \frac{\phi_{i j}\left(H_{i}\right)+H_{j}}{H_{j}}
$$

Therefore we have for each $i, j$ :

$$
\left|\frac{\phi_{i j}\left(H_{i}\right)+H_{j}}{H_{j}}\right| \leq\left|\frac{\phi(H)+H}{H}\right| \leq \prod_{i, j=1}^{n}\left|\frac{\phi_{i j}\left(H_{i}\right)+H_{j}}{H_{j}}\right| .
$$

The proof follows now easily. In particular, the last statement follows from the fact that $\left|\frac{\phi_{i j}\left(H_{i}\right)+H}{H}\right|$ divides $\left|\frac{\phi(H)+H}{H}\right|$.

Corollary 2.7. Let $G=G_{1} \oplus G_{2}$ be a group and $H$ be a uniformly fully inert subgroup of $G$ with inertial bound n. Then:

(a) $H \cap G_{2}$ is a uniformly fully inert subgroup of $G_{2}$ with inertial bound at most $n$;

(b) if $\psi: G \rightarrow G_{1}$ is a homomorphism such that $\psi(H)$ is infinite, then $H \cap G_{1}$ is infinite as well.

Proof. Part (a) follows from Corollary 2.5 and Proposition 2.6. Concerning (b), assume, by way of contradiction, that $H \cap G_{1}$ is finite. Thus, $H \cap \psi(H) \leq H \cap G_{1}$ is finite as well, and since $\psi(H)$ is infinite, we deduce that $\psi(H) /(H \cap \psi(H))$ is infinite, a contradiction.

The next result considers the case when $G$ is the direct sum of two fully invariant subgroups; it will be the main tool in the proof of Theorem 5.7 .

Proposition 2.8. Let $G=G_{1} \oplus G_{2}$ be a group which is the direct sum of two fully invariant subgroups. If $H$ is a uniformly fully inert subgroup of $G$ with inertial bound $\mathbf{i b}(H)=n$, and $H_{i}(i=1,2)$ are the projections of $H$ in $G_{i}$, then

(1) the subgroups $H_{i}$ are uniformly fully inert with inertial bounds $\mathbf{i b}\left(H_{i}\right)$ dividing $n$;

(2) if furthermore $n \cdot i d_{G_{2}} \in \operatorname{End}\left(G_{2}\right)$ is an automorphism of $G_{2}$, then:

(2a) $H=H_{1} \oplus H_{2}$;

$\left(2_{b}\right) H_{2}$ is a fully invariant subgroup of $G_{2}$;

(2c) $H \in \operatorname{Jn} \tilde{v}(G)$ if and only if $H_{1} \in \operatorname{Jn} \tilde{v}\left(G_{1}\right)$;

$\left(2_{d}\right) H \in \mathcal{J} n v(G)$ if and only if $H_{1} \in \operatorname{Jn} v\left(G_{1}\right)$.

Proof. (1) follows from Proposition 2.6. 
(2a) Let $\pi_{i}: G \rightarrow G_{i}(i=1,2)$ be the projections, so that $H_{i}=\pi_{i}(H)$. In order to to prove that $H=H_{1} \oplus H_{2}$, it suffices to prove that $H \geq \pi_{2}(H)$. Pick $h=h_{1}+h_{2} \in H \leq H_{1} \oplus H_{2}$, where $h_{i}=\pi_{i}(h)$. To prove that $h_{2} \in H$, let $\xi \in \operatorname{End}\left(G_{2}\right)$ be the inverse of $n \cdot i d_{G_{2}} \in \operatorname{End}\left(G_{2}\right)$; extend $\xi$ to an endomorphism $\alpha$ of $G$ by sending $G_{1}$ to zero. As $h_{2}=n \alpha(h) \in n \alpha(H) \subseteq H$, we are done.

$\left(2_{b}\right)$ Since $n \cdot i d_{G_{2}}$ is invertible in $\operatorname{End}\left(G_{2}\right)$ and it belongs to the center of that ring, the fact that $n \phi\left(H_{2}\right) \leq H_{2}$ for every endomorphism $\phi$ of $G_{2}$ implies that $H_{2}$ is a fully invariant subgroup of $G_{2}$.

(2c) Assume that $H_{1} \in \mathcal{J} n \tilde{v}\left(G_{1}\right)$, so there exists a fully invariant subgroup $L \leq G_{1}$ such that $H_{1} \sim L$ ( $H$ is commensurable with $L)$. By our assumption on $G$ and items $(2 a)$ and $\left(2_{b}\right)$, it follows that the subgroup $L \oplus H_{2}$ of $G$ is fully invariant. Since obviously $H=H_{1} \oplus H_{2} \sim L \oplus H_{2}$, we are done.

$\left(2_{d}\right)$ Argue similarly to $\left(2_{c}\right)$.

\subsection{The fully invariant hull and the fully invariant core}

Every subgroup $H$ of a group $G$ is sandwiched between the minimal fully invariant subgroup $H^{\star}$ of $G$ containing it, and the maximal fully invariant subgroup $H_{\star}$ contained in it. Here $H^{\star}$ is the intersection of all fully invariant subgroups containing $H$ and $H_{\star}$ is the sum of all fully invariant subgroups contained in $H$. The subgroups $H^{\star}$ and $H_{\star}$ are called the fully invariant hull and the fully invariant core of $H$, respectively.

It seems to be quite natural to consider the subgroups $H_{\star}$ and $H^{\star}$ as candidates for a test of whether $H$ is commensurable with some fully invariant subgroup, i.e., $H \in J n \tilde{v^{2}}(G)$. Indeed, the conditions $H^{\star} / H$ finite or $H / H_{\star}$ finite are sufficient to ensure that $H \in \mathcal{J} n \tilde{v^{2}}(G)$. Thus it is natural to consider the two families of subgroups of $G$ :

$$
\operatorname{Inv}{ }^{\star}(G)=\left\{H \in \mathcal{L}(G):\left|H^{\star} / H\right|<\infty\right\} \text { and } \operatorname{In} v_{\star}(G)=\left\{H \in \mathcal{L}(G):\left|H / H_{\star}\right|<\infty\right\} .
$$

Lemma 2.9. The sets $\operatorname{In} v^{\star}(G)$ and $\operatorname{In} v_{\star}(G)$ are sublattices of $\operatorname{In} v^{\sim}(G)$ containing $\operatorname{Inv}(G)$, hence

$$
\operatorname{Jn} v(G) \subseteq \operatorname{Jn} v^{*}(G) \subseteq \operatorname{Jn} \tilde{v^{2}}(G) \text { and } \operatorname{Jn} v(G) \subseteq \operatorname{J} n v^{*}(G) \subseteq \operatorname{Jn} \tilde{v^{*}(G)} .
$$

Proof. The inclusions $\operatorname{In} v(G) \subseteq \operatorname{Jn} v^{\star}(G)$ and $\operatorname{In} v(G) \subseteq \operatorname{Jn} v_{\star}(G)$ are obvious. Note that, if $H$ if a subgroup of a group $G$ with either $\left|H / H_{\star}\right|<\infty$ or $\left|H^{*} / H\right|<\infty$, then $H \in \operatorname{J} n \tilde{v}(G)$. Furthermore, if $H, K \in \operatorname{J} n v_{*}(G)$, then we have:

$$
\left|(H \cap K) /\left(H_{\star} \cap K_{\star}\right)\right|=\left|(H \cap K) /\left(H \cap K_{\star}\right)\right| \cdot\left|\left(H \cap K_{\star}\right) /\left(H_{\star} \cap K_{\star}\right)\right| \leq\left|K / K_{\star}\right| \cdot\left|H / H_{\star}\right|<\infty
$$

and, dually, writing + in place of $\cap$ we have

$$
\left|(H+K) /\left(H_{\star}+K_{\star}\right)\right|=\left|(H+K) /\left(H+K_{\star}\right)\right| \cdot\left|\left(H+K_{\star}\right) /\left(H_{\star}+K_{\star}\right)\right| \leq\left|K / K_{\star}\right| \cdot\left|H / H_{\star}\right|<\infty .
$$

So $J n v_{\star}(G)$ is a sublattice of $\operatorname{Inv}(G)$. The proof that $J n v^{\star}(G)$ is a sublattice of $J n \tilde{v^{*}}(G)$ is obtained in the same way as above, by writing $H^{\star}, K^{\star}$ in place of $H, K$ and $H, K$ in place of $H_{\star}, K_{\star}$.

The next lemma will be used in the sequel (see Lemma 5.1).

Lemma 2.10. If $H$ is a uniformly fully inert subgroup of the group $G$ with inertial bound $\mathbf{i b}(H)=n$, then

$$
n H^{\star} \leq H_{\star} \leq H \leq H^{\star} .
$$

Hence, both $H^{\star} / H$ and $H / H \star$ are bounded by $n$.

Proof. For each $\phi \in \operatorname{End}(G)$, all subgroups $\phi H$ have order dividing $n$ modulo $H$, hence $H^{\star} / H$ is bounded by $n$ and consequently $n H^{\star} \leq H$. Also $n H^{\star}$ is fully invariant and $n H^{\star} \leq H$, hence $n H^{\star} \leq H_{\star}$. Thus $n H^{\star} \leq H$ and $n H \leq H_{\star}$, so also $H / H_{\star}$ is bounded by $n$.

Two natural questions concerning the sublattices $\mathfrak{I n} v^{\star}(G)$ and $\mathfrak{I} n v_{\star}(G)$ related to the sublattice $\mathcal{J}_{u}(G)$ arise.

Question 2.11. Let $H$ be a subgroup of a group $G$. 
(I) Does $H \in \mathcal{J}_{u}(G)$ imply $H \in \mathcal{J}_{n} v^{\star}(G)$, i.e., $\left|H^{\star} / H\right|<\infty$ ?

(II) Does $H \in \mathcal{J}_{u}(G)$ imply $H \in \mathcal{J}_{n v \star}(G)$, i.e., $\left|H_{\star} / H\right|<\infty$ ?

Similar questions can be posed replacing $\mathcal{J}_{u}(G)$ by $J n v^{\sim}(G)$ and $\mathcal{J}(G)$. Our interest in these question stems from the simple observation that a positive answer to any of these two items would imply that $H \in \operatorname{In} \tilde{\sim}(G)$, according to Lemma 2.9, i.e., our main conjecture is "locally" solved, as far as the specific subgroup $H$ is concerned. In case the answer is positive for all subgroups $H$ of $G$, then $G$ satisfies our main conjecture.

We will see in Section 5 that the answer to Question 2.11 is positive for some subgroups $H$ of $G$ for a large classe of abelian groups $G$. In particular, it is true for all subgroups of torsion-free groups $G$ of finite rank. However, in general the answer is in the negative, as the next examples show.

The first (easier) example shows that $\operatorname{In} v^{\star}(G) \neq \operatorname{J} n v^{\sim}(G) \neq J n v_{\star}(G)$.

Example 2.12. Let $G=\bigoplus_{i=0}^{\infty}\left\langle e_{i}\right\rangle$ with $o\left(e_{i}\right)=p$ for all $i$.

(a) if $H=\left\langle e_{1}\right\rangle$, then $H_{\star}=0$, so that $H \sim H_{\star}$. On the other hand, $H^{\star}=G$, so that $H^{\star} / H$ is infinite. Therefore, $H \in \mathcal{J} n v_{*}(G)$, but $H \notin J n v^{\star}(G)$.

(b) if $H=\bigoplus_{i=1}^{\infty}\left\langle e_{i}\right\rangle$, then $H^{\star}=G$ and $H_{\star}=0$. Therefore, $H \sim H^{\star}$ while $H / H_{\star}=H$ is infinite, i.e., $H \in \operatorname{Jn} v^{\star}(G)$, but $H \notin J n v_{*}(G)$.

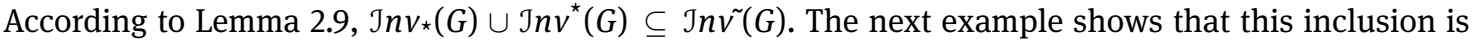
proper as well.

Example 2.13. Let

$$
G=\bigoplus_{i \geq 0}\left\langle e_{i}\right\rangle \oplus \bigoplus_{j \geq 0}\left\langle f_{j}\right\rangle \simeq \bigoplus_{\aleph_{0}} \mathbb{Z}(p) \oplus \bigoplus_{\aleph_{0}} \mathbb{Z}\left(p^{2}\right),
$$

where $o\left(e_{i}\right)=p$ and $o\left(f_{j}\right)=p^{2}$ for each $i, j \in \mathbb{N}$. Then the subgroup

$$
H=\bigoplus_{i \geq 1}\left\langle e_{i}\right\rangle \oplus\left\langle f_{1}\right\rangle \oplus \bigoplus_{j \geq 2}\left\langle p f_{j}\right\rangle
$$

is commensurable with the socle

$$
G[p]=\bigoplus_{i \geq 0}\left\langle e_{i}\right\rangle \oplus \bigoplus_{j \geq 0}\left\langle p f_{j}\right\rangle
$$


Corollary 2.2.

We show now that $H^{\star}=G$ and $H_{\star}=0$, so that both $H^{\star} / H=G / H$ and $H / H_{\star}=H$ are infinite, i.e., the uniformly fully inert subgroup $H$ simultaneously fails to satisfy both $(I)$ and $(I I)$ in Question 2.11. To see that $H^{\star}=G$ note that $f_{1}$ can be sent by appropriate endomorphisms of $G$ to any other element of $G$; so $H^{\star}=G$. To check that $H_{\star}=0$ assume, by way of contradiction, that $0 \neq x \in H_{\star}$. If $x$ has order $p^{2}$, it generates a cyclic summand, so it can be sent to $e_{0} \notin H$ (or to $f_{0} \notin H$ ) by an appropriate endomorphism of $G$, contradicting the full invariance of $H_{\star}$ and the inclusion $H_{\star} \leq H$. If $x$ has order $p$, either it has height 0 , in which case it generates a cyclic summand, so it can be sent to $e_{0} \notin H$, or $x=p y$. In the latter case $y$ generates a cyclic summand and can be sent to $f_{0}$ by an appropriate endomorphism $\phi$ of $G$. Hence, $\phi(x)=\phi(p y)=p f_{0} \notin H$, again a contradiction.

As mentioned above, the subgroup $H$ in Example 2.13 is commensurable with a fully invariant subgroup of $G$ (namely, $G[p]$ ). More precisely, it was proved in [24] that every fully inert subgroup of a direct sum of cyclic $p$-groups has this property. Actually, the proof of this fact in [24] produces in the general case a fully invariant commensurable subgroup smaller than $H^{\star}$ or $H_{\star}$. Another similar example is given in [24, Example 3.11].

Let us see now a further example which has an impact in the non-abelian context. 
Example 2.14. Let $G=\bigoplus_{i \in \mathbb{Z}}\left\langle a_{i}\right\rangle$ with $\left\langle a_{i}\right\rangle \simeq \mathbb{Z}\left(p^{2}\right)$ and $H=\left\langle a_{0}\right\rangle \oplus \bigoplus_{i \neq 0,1}\left\langle p a_{i}\right\rangle$. Then clearly $H$ is commensurable with $G[p]=p G=\bigoplus_{i \in \mathbb{Z}}\left\langle p a_{i}\right\rangle$ the socle of $G$. Let $\phi$ be the automorphism of $G$ such that $\phi\left(a_{i}\right)=a_{i+1}$ for each $i$ (Bernoulli shift). Then, for all $j$, we have $a_{j}=\phi^{j}\left(a_{0}\right) \in \phi^{j}(H) \leq H^{\star}$ hence $H^{\star}=G$.

Therefore, $H^{\star} / H$ is infinite and the same occurs to $H / H_{\star}$. In fact, since $\phi$ is invertible, we must have $H_{\star}=$ $\phi^{j}\left(H_{\star}\right)$ for all $j$ and clearly $\phi^{j}\left(H_{\star}\right) \leq \phi^{j}(H)$. The elements in $\phi^{j}(H)$ have $(j+1)$-component equal 0 . Thus $H_{\star} \leq \bigcap_{j} \phi^{j}(H)=\{0\}$ so that $H / H_{\star}$ is infinite.

Finally, the subgroup $G^{-}=\bigoplus_{-i \in \mathbb{N}}\left\langle a_{i}\right\rangle$ is $\phi$-inert but not uniformly fully inert, as

$$
\left|\phi^{n}\left(G^{-}\right) / \phi^{n}\left(G^{-}\right) \cap G^{-}\right|=\left|\phi^{n}\left(G^{-}\right) / G^{-}\right|=p^{n}
$$

for each $n \in \mathbb{N}$.

Remark 2.15. Turning back to the non-abelian context, we note that Definitions 1.1, 1.2, 1.3, 1.5, Proposition 2.1, Lemmas 2.3, 2.4 and Corollary 2.5 can be easily formulated or carried out in this general context.

(a) In the language of non-commutative groups, the argument of Example 2.14 proves exactly that in the wreath product $\mathbb{Z}\left(p^{2}\right) \imath \mathbb{Z} \simeq G \rtimes\langle\phi\rangle$ a subgroup may be commensurable with a normal subgroup even if it is not commensurable neither with its normal closure nor with its normal core. Moreover, the subgroup $G^{-}$is inert in $G$ (in Belyaev's terminology) but not uniformly inert (for a similar example see also [5], where a quotient of this group is considered).

(b) The paper [4] provides an example of a nilpotent $p$-group $G$ with the property that each subgroup of $G$ is commensurable with a normal subgroup even if there are subgroups $H$ of $G$ which do not have the property that $\left|H: H_{G}\right|$ is finite and also subgroups $H$ which do not have the property that $\left|H^{G}: H\right|$ is finite. Here, as usual, $H_{G}$ (resp. $H^{G}$ ) denotes the normal core (resp. normal closure) of $H$ in $G$.

\section{Known facts for divisible groups and completely decomposable groups}

\subsection{The chain $\left({ }^{\star}\right)$ for divisible groups}

We consider now the chain $\left.{ }^{\star}\right)$ of sublattices of $\mathcal{L}(G)$ when $G$ is a divisible group. Fully inert subgroups of divisible group have been studied in [16] and some more details have been furnished in [8]. From [8, Proposition 5.6] and [16, Theorem 4.9] we get the following

Example 3.1. For the torsion-free divisible group of finite rank $\mathbb{Q}^{n}$ the following holds:

$$
\left\{\{0\}, \mathbb{Q}^{n}\right\}=\operatorname{Inv}\left(\mathbb{Q}^{n}\right)=\operatorname{Inv} \sim\left(\mathbb{Q}^{n}\right)=\mathcal{J}_{u}\left(\mathbb{Q}^{n}\right) \subsetneq \mathcal{J}\left(\mathbb{Q}^{n}\right)
$$

and $\mathcal{J}\left(\mathbb{Q}^{n}\right)$ consists of the homogeneous completely decomposable groups of rank $n$, i.e., of those subgroups of the form $H_{1} \oplus \ldots \oplus H_{n}$, where the $H_{i}$ 's are all isomorphic to a fixed subgroup of $\mathbb{Q}$.

More generally, for divisible groups the picture is the following.

Theorem 3.2. [8, Theorem 5.13, Corollary 5.14] Let $D$ be a divisible group. Then $\operatorname{In} v^{\sim}(D)=\operatorname{In} v^{\sim}(t(D)) \cup\{D\}=$ $\mathcal{J}_{u}(D)$. Furthermore, $\mathcal{J}_{u}(D)=\mathcal{J}(D)$ if and only if either $r_{0}(D)=0$, or $r_{0}(D)=\infty$.

In other words, for a divisible group $D$ the following are equivalent:

$$
0<r_{0}(D)<\infty \quad \Leftrightarrow \quad \mathfrak{J n} \tilde{v}(D) \subsetneq \mathcal{J}(D) \quad \Leftrightarrow \quad \mathcal{J}_{u}(D) \subsetneq \mathcal{J}(D)
$$

and in all cases $\operatorname{In} v^{\sim}(D)=\mathcal{J}_{u}(D)$, thus supporting Conjecture 1.6. 


\subsection{The chain ( $\left.{ }^{\star}\right)$ for completely decomposable groups}

A completely decomposable group is a torsion-free group which is isomorphic to a direct sum of rational groups, i.e., of subgroups of the rational group $\mathbb{Q}$. We refer to [21, Chapter 12, Section 1] for the basic notions of characteristic and type and for their properties, and for the fundamental fact proved by Baer that the types are in bijection with the isomorphism classes of the rational groups, thus providing their classification.

Here we recall just few things. A characteristic is a sequence of non-negative integers or symbols $\infty$ indexed by the prime numbers

$$
\chi=\left(h_{2}, h_{3}, \ldots, h_{p}, \ldots\right) .
$$

Two characteristics are declared equivalent if they have the same $\infty$ 's and finitely many differences in the other entries. A type is the equivalence class of a characteristic with respect to this equivalence relation (our notation is slightly different from that in [21], where different brackets are used):

$$
\mathbf{t}=[\chi]=\left[h_{2}, h_{3}, \ldots, h_{p}, \ldots\right] .
$$

Given two characteristics $\chi_{1}=\left(k_{p}\right)_{p}$ and $\chi_{2}=\left(h_{p}\right)_{p}$ and the two corresponding types $\mathbf{t}_{1}$ and $\mathbf{t}_{2}$, their products are defined by

$$
\begin{aligned}
\chi_{1} \cdot \chi_{2} & =\left(k_{2}+h_{2}, k_{3}+h_{3}, \ldots, k_{p}+h_{p}, \ldots\right) \\
\mathbf{t}_{1} \cdot \mathbf{t}_{2} & =\left[k_{2}+h_{2}, k_{3}+h_{3}, \ldots, k_{p}+h_{p}, \ldots\right]
\end{aligned}
$$

where $\infty+n=\infty$ and $\infty+\infty=\infty$. The inequality $\chi_{1} \geq \chi_{2}$ is defined pointwise; accordingly, $\mathbf{t}_{1} \geq \mathbf{t}_{2}$ for the types means that the same inequality holds for two suitable characteristics representing them. With these orders the characteristics form a complete distributive lattice, while the types form a non-complete distributive lattice (e.g., the sequence of types $\mathbf{t}_{n}=[n, n, \ldots, n, \ldots](n \geq 1)$ has no supremum).

If $\mathbf{t}_{1} \geq \mathbf{t}_{2}$ and $\chi_{1} \geq \chi_{2}$ represent these types, the colon of the two types is given by

$$
\mathbf{t}_{1}: \mathbf{t}_{2}=\left[k_{2}-h_{2}, k_{3}-h_{3}, \ldots, k_{p}-h_{p}, \ldots\right]
$$

where $\infty-n=\infty$ and $\infty-\infty=\infty$.

The type $\mathbf{t}=\left[h_{2}, h_{3}, \ldots, h_{p}, \ldots\right]$ is idempotent if, for every $p$, either $h_{p}=0$ or $h_{p}=\infty$. The reduced type $\mathbf{t}_{0}$ of the type $\mathbf{t}$ is obtained from $\mathbf{t}$ replacing all the $h_{p}$ 's different from $\infty$ by 0 ; obviously $\mathbf{t}_{0}=\mathbf{t}: \mathbf{t}$.

If $G$ is a torsion-free group, the characteristic (or height sequence) of an element $a \in G$ is the sequence of the $p$-heights of $a$ :

$$
\chi(a)=\left(h_{2}(a), h_{3}(a), \ldots, h_{p}(a), \ldots\right)
$$

where $h_{p}(a)$ is the maximal $k \in \mathbb{N}$ such that $a \in p^{k} G$, if such a $k$ does exist, or $\infty$ otherwise. The type $\mathbf{t}(a)$ of $a$ is the type represented by $\chi(a)$.

The type $\mathbf{t}(A)$ of a rational group $A$ is, by definition, the type $\mathbf{t}(a)$ of any $0 \neq a \in A$. If $A \leq B$ are rational groups, then $\mathbf{t}(A) \leq \mathbf{t}(B)$ and $\mathbf{t}_{0}(A) \leq \mathbf{t}_{0}(B)$.

Given two rational groups $A$ and $B, \operatorname{Hom}(A, B)$ is naturally isomorphic to a rational group and $\mathbf{t}(A) \not \leq \mathbf{t}(B)$ implies $\operatorname{Hom}(A, B)=0$, while $\mathbf{t}(A) \leq \mathbf{t}(B)$ implies $\mathbf{t}(\operatorname{Hom}(A, B))=\mathbf{t}(A): \mathbf{t}(B)$.

For every rational group $A$ of type $\mathbf{t}$, the endomorphism ring $\operatorname{Hom}(A, A)$ is isomorphic to $A_{0}$, the rational group of reduced type $\mathbf{t}_{0}$, and $\left.\operatorname{Hom}\left(A_{0}, A\right)\right) \cong A$. From this fact it is easy to deduce the behavior of the chain $\left({ }^{\star}\right)$ for rational groups. We include detailed proofs as a prelude to the next results on completely decomposable groups. In particular, Proposition 3.3 should be compared with Corollary 3.5 .

Proposition 3.3. Let $H$ be a non-zero subgroup of a rational group $G$. Then

(1) $H \in \mathcal{J}(G)$, i.e., all non-zero subgroups are fully inert in $G$;

(2) $H \in \operatorname{Jnv}(G)$ if and only if $\mathbf{t}_{0}(G)=\mathbf{t}_{0}(H)$, i.e., $p G=G$ implies $p H=H$;

(3) $\operatorname{Jn} v(G)=\operatorname{Jn} v \widetilde{\sim}(G)=\mathcal{J}_{u}(G)$;

(4) $\operatorname{Inv}(G)=\mathcal{J}(G)$ if and only if $p G \neq G$ for all $p$, i.e., the type $\mathbf{t}(G)$ contains no $\infty$ 's. 
Proof. Recall that an endomorphism $\phi$ of $G$ is induced by the multiplication by a rational number $m / n$ with $(m, n)=1$, where $n$ is a product of primes $p$ such that $G=p G$, since $\operatorname{Hom}(G, G) \cong G_{0}$ as rings.

(1) Let $\phi: G \rightarrow G$ be as above. Then $(H+\phi H) / H \cong(n H+m H) / n H$; being $m, n$ coprime, $H=n H+m H$, therefore $(H+\phi H) / H \cong H / n H$, which is finite.

(2) First we note that $\mathbf{t}_{0}(G)=\mathbf{t}_{0}(H)$ precisely when $p G=G$ implies $p H=H$, as the inequality $\mathbf{t}_{0}(H) \leq \mathbf{t}_{0}(G)$ is always true.

If $\mathbf{t}_{0}(H) \neq \mathbf{t}_{0}(G)$, i.e., $p G=G$ and $p H<H$ for some prime $p$, then the endomorphism $\phi$ of $G$ induced by the multiplication by $1 / p$ does not satisfy $\phi H \leq H$, so $H$ is not fully invariant. Conversely, assume that $p H=H$ for all $p$ such that $p G=G$ and let $\phi: G \rightarrow G$ be as above. The equality $n H=H$ clearly implies that $\phi H \leq H$, so $H$ is fully invariant.

(3) Assume that $H \in \mathcal{J}_{u}(G)$. According to (2), to prove that $H \in \operatorname{Jn} v(G)$ we have to check that $\mathbf{t}_{0}(G)=\mathbf{t}_{0}(H)$. Assume for a contradiction that $p G=G$ and $p H<H$ for some prime $p$. Then the endomorphism $\psi_{n}$ of $G$ induced by the multiplication by $1 / p^{n}$ satisfies $\left(H+\psi_{n} H\right) / H \cong H / p^{n} H$, whose cardinality tends to $\infty$.

(4) From (1) and (2) we get that $\operatorname{Jn} v(G)=\mathcal{J}(G)$ if and only, for every non-zero subgroup $H$ of $G, p G=G$ implies $p H=H$. But if $p G=G, G$ certainly contains subgroups $H$ such that $p H<H$, so $p G \neq G$ for all $p$.

In the sequel we address the class of completely decomposable groups. For general information on these groups we refer to [21, Chapter 12, Section 3].

The following theorem concerning the chain $\left({ }^{\star}\right)$ for completely decomposable groups of finite rank combines results from three papers, namely [6-8] (note, that (1) $\Rightarrow(3)$ is trivial, while (3) $\Rightarrow$ (4) holds according to Proposition 2.1). First, Chekhlov [6, Theorem 2] established the equivalence of (2) and (3). This triggered the equivalence (2) $\Leftrightarrow(3) \Leftrightarrow$ (4) proved in [8, Theorem 5.10]. Recently, Chekhlov [7, Theorem 1] proved that the conditions (2) and (3) are equivalent to (1).

Theorem 3.4. For a completely decomposable group of finite rank $G=G_{1} \oplus \ldots \oplus G_{n}$, where $G_{i}$ has rank 1 for each $i=1, \ldots, n$, the following are equivalent:

1. each fully inert subgroup $H$ of $G$ has finite index in its fully invariant hull $H^{\star}$, i.e., $\mathcal{J}(G)=\operatorname{Inv}{ }^{\star}(G)$;

2. $\mathbf{t}\left(G_{i}\right)$ contains no $\infty$ for each $i$, and two types $\mathbf{t}\left(G_{i}\right)$ and $\mathbf{t}\left(G_{j}\right)$ are either equal or incomparable;

3. $\mathcal{J}(G)=\mathcal{J} n \tilde{v}(G)$, i.e., every fully inert subgroup is commensurable with a fully invariant subgroup;

4. $\mathcal{J}(G)=\mathcal{J}_{u}(G)$, i.e., every fully inert subgroup is uniformly fully inert.

It follows from this theorem that a uniformly fully inert subgroup $H$ of a completely decomposable groups of finite rank has finite index in its fully invariant hull $H^{\star}$. In Corollary 5.3 we will obtain the notably stronger result that this holds for an arbitrary torsion-free group of finite rank.

Let us recall also the concluding result for homogeneous completely decomposable groups $G=A_{1} \oplus \ldots \oplus$ $A_{n}$ of finite rank, defined by the property that the subgroups $A_{i}$ are all isomorphic; their common type is then the type $\mathbf{t}(G)$ of $G$.

Corollary 3.5. [6, Corollary 3] Let $G$ be a homogeneous completely decomposable group of finite rank. Then:

1. a fully inert subgroup $H$ of $G$ is commensurable with a fully invariant subgroup of $G$ if and only if $p H=H$ for every prime $p$ such that $p G=G$;

2. every fully inert subgroup of $G$ is commensurable with a fully invariant subgroup of $G$ if and only if $p G \neq G$ for every prime $p$, i.e., $\mathbf{t}(G)$ contains no $\infty$.

Note that Corollary 3.5 extends Proposition 3.3 for homogeneous completely decomposable groups from rank 1 to arbitrary finite rank. While (1) is an immediate corollary of the equivalence of Theorem 3.4, item (2) provides something that goes beyond that result, as one finds a complete description of the family $\operatorname{In} \tilde{v}(G)$.

Recently Chekhlov extended in [7] Theorem 3.4 to completely decomposable groups of arbitrary rank, but with only a finite number of homogeneous components (i.e., the maximal homogeneous summands). First he showed that it is enough to consider reduced completely decomposable groups, and for these groups he proved the following 
Theorem 3.6. [7, Theorem 2] Assume that $G=G_{1} \oplus \ldots \oplus G_{n}$ is a reduced completely decomposable group, where $G_{i}$ is a homogenous component for each $i=1, \ldots, n$. Then the following are equivalent:

1. $\mathcal{J}(G)=\operatorname{In} v^{\sim}(G)$, i.e. every fully inert subgroup of $G$ is commensurable with a fully invariant subgroup;

2. if a homogeneous component $G_{i}$ has finite rank, then:

(a) $\mathbf{t}\left(G_{i}\right)$ contains no $\infty$;

(b) if $\mathbf{t}\left(G_{i}\right)<\mathbf{t}\left(G_{j}\right)$ for some j, then $r k_{0}\left(G_{j}\right) \geq \aleph_{0}$;

(c) under the hypothesis of (b), if there are no types $\mathbf{t}\left(G_{k}\right)$ between $\mathbf{t}\left(G_{i}\right)$ and $\mathbf{t}\left(G_{j}\right)$, the type $\mathbf{t}\left(G_{j}\right): \mathbf{t}\left(G_{i}\right)$ is idempotent.

We conjecture that one may add to the equivalent conditions of Theorem 3.6 also the equality $\mathcal{J}(G)=\mathcal{J}_{u}(G)$, i.e., every fully inert subgroup is uniformly fully inert (see Question 7.4 at the end of the paper).

\section{Orsatti groups, groups with finite ranks and narrow groups}

In this section we introduce three classes of abelian groups which are strictly related to each other, namely, the Orsatti groups introduced in [28], the narrow groups defined in [15], and the groups with finite ranks already defined in the Introduction.

In the sequel we make use of a functorial topology, the so called natural topology, or $\mathbb{Z}$-topology, according to some authors (see for more details and background [21, 27]). It is defined as follows: for an abelian group $G$ the natural topology topology $v_{G}$ has as basic neighborhoods of 0 the subgroups $n G=\{n g \mid g \in G\}$, running $n$ over the set of all positive integers. Clearly, $v_{G}$ is Hausdorff precisely when the first Ulm subgroup $G^{1}=\bigcap_{n>0} n G$ of $G$ is trivial. It is easy to see that this is equivalent to asking $G$ to be residually finite [14].

Orsatti investigated in [28] the class of abelian groups $G$ such that $\left(G, v_{G}\right)$ is compact. These groups are named Orsatti groups in [12, 20], where the class of these groups is denoted by $\mathfrak{O}$. The description of Orsatti groups $G$, obtained in [28], is as follows:

$$
G=\prod_{p} \mathbb{J}_{p}^{k_{p}} \oplus F_{p},
$$

where $\mathbb{J}_{p}$ denotes the compact group of $p$-adic integers, $k_{p} \in \mathbb{N}$ and $F_{p}$ is a finite abelian $p$-group. In other words, $G$ is a direct product of finitely generated $\mathbb{J}_{p}$-modules, for $p \in \mathbb{P}$. It is immediate to verify that an Orsatti group $G$ satisfies the property that $G / n G$ is finite for every integer $n$. The Orsatti groups have also the following remarkable property established in [28] under the assumption of the Generalized Continuum Hypothesis: they are precisely the abelian groups $G$ that admit a unique compact group topology, namely $v_{G}$. Since $v_{G}$ is functorial, every $\phi \in \operatorname{End}(G)$ is automatically continuous.

Inspired by Orsatti's definition of the class $\mathfrak{O}$, one can consider the class of abelian groups $G$ satisfying the property that $G / n G$ is finite for every integer $n$; in topological terms, $\left(G, v_{G}\right)$ is totally bounded. It is well known that for residually finite groups this amounts to say that the completion of $\left(G, v_{G}\right)$ is compact. These groups are named according to the following

Definition 4.1. [15] An abelian group $G$ is said to be narrow whenever $G / n G$ is finite for every natural number $n>0$. We denote by $\mathfrak{N}$ the class of all narrow groups.

The class $\mathfrak{N}$ of narrow groups was introduced in [15] as an example of a large class of abelian groups $G$ such that every endomorphism of $G$ has adjoint algebraic entropy zero, and was also highlighted in [14]. The adjoint algebraic entropy is the natural dual of the algebraic entropy for endomorphisms of abelian groups, deeply investigated in $[13,18]$ (see also the survey [23]). In [15] the following characterizations of narrow groups are presented, involving the family $\mathcal{C}(G)$ of all subgroups of finite index of $G$.

Theorem 4.2. [15, Theorem 3.3] For an abelian group $G$ the following conditions are equivalent:

(a) G is narrow; 
(b) $G / p G$ is finite for every prime number $p$;

(c) the family $\mathrm{e}(G)$ is countable;

(d) $|\mathfrak{C}(G)|<2^{\aleph_{0}}$;

(e) $\mathcal{C}(G)$ contains a countable decreasing cofinal chain;

(f) the natural topology $v_{G}$ of $G$ coincides with the profinite topology of $G$.

In the next theorem we provide some more properties of the narrow groups. In particular, it gives various reduction to relevant classes of groups, as reduced groups, torsion and torsion-free groups.

Theorem 4.3. The following facts hold for the class $\mathfrak{N}$ of narrow groups.

(a) $\mathfrak{N}$ is closed under taking quotients, pure subgroups and extensions.

(b) $\mathfrak{N}$ contains the class of divisible groups and the class of Orsatti groups; moreover $G \in \mathfrak{N}$ if and only if $G / d(G) \in \mathfrak{N}$.

(c) $G \in \mathfrak{N}$ if and only if $G / G^{1} \in \mathfrak{N}$.

(d) $G \in \mathfrak{N}$ if and only if both $t(G)$ and $G / t(G)$ belong to $\mathfrak{N}$.

(e) If $G$ is a reduced torsion group, then $G \in \mathfrak{N}$ if and only if $r_{p}(G)<\infty$ for all primes $p$, equivalently, all p-primary components are finite.

(f) $\mathfrak{N}$ contains the groups with finite ranks;

(g) If $G$ is a reduced torsion-free group, then $G \in \mathfrak{N}$ if and only if it is a pure subgroup of a compact group of the form $\prod_{p} \mathbb{J}_{p}^{k_{p}}$, where the $k_{p}$ 's are non-negative integers.

Proof. (a) Assume $G \in \mathfrak{N}, H \leq G$ and $n \in \mathbb{Z}$. Then $(G / H) / n(G / H) \cong G /(n G+H)$, which is an epic image of $G / n G$, so it is finite, hence $G / H \in \mathfrak{N}$.

If $H$ is pure in $G$, then $H / n H=H /(H \cap n G) \cong(H+n G) / n G$, which is finite as a subgroup of $G / n G$, hence also $H \in \mathfrak{N}$.

Finally, consider the exact sequence $0 \rightarrow A \rightarrow B \rightarrow B / A \rightarrow 0$, where $A, B / A \in \mathfrak{N}$. It gives rise the exact sequence

$$
0 \rightarrow(A+n B) / n B \rightarrow B / n B \rightarrow B /(A+n B) \rightarrow 0,
$$

where $(A+n B) / n B \cong A /(A \cap n B)$ is finite, as an epic image of $A / n A$, and $B /(A+n B) \cong(B / A) / n(B / A)$ is also finite. Hence also $B \in \mathfrak{N}$.

(b) If $D$ is a divisible group, then $D=n D$ for all $n \in \mathbb{Z}$, hence trivially $D \in \mathfrak{N}$. This makes the final assertion obvious, as the class $\mathfrak{N}$ is closed under taking finite direct sums and quotients, according to item (a). To see that an Orsatti group belong to $\mathfrak{N}$ it is enough to consider the local case, which immediately follows from the fact that $\mathbb{J}_{p} / p^{k} \mathbb{J}_{p}$ is finite for all $k \geq 0$.

(c) is trivial in view of (a), as $G / n G \cong\left(G / G_{1}\right) /\left(n G / G_{1}\right)$ for all $n \in \mathbb{Z}$.

(d) follows from item (a), taking into account that $t(G)$ is pure in $G$.

(e) Write $G=\bigoplus t_{p}(G)$, the primary decomposition of $G$. For each $n \in \mathbb{Z}, G / n G$ is a quotient of a direct sum of finitely many $t_{p}(G)$ 's. Thus, using (a), we deduce that $G \in \mathfrak{N}$ if and only if $t_{p}(G) \in \mathfrak{N}$ for all primes $p$. So we can assume that $G$ is a reduced $p$-group for some prime $p$. Pick a basic subgroup $B$ of $G$. If $G \in \mathfrak{N}$, then $G / p G \cong B / p B$ is finite, henceforth $B$, as a direct sum of cyclic $p$-groups, must be finite, and consequently $G=B$ is finite. The converse is trivial.

(f) Making use of item (d), we can consider separately the cases when $G$ is torsion-free and when $G$ is torsion. Moreover, since the class of groups of finite rank is closed under taking subgroups, we can assume, in view of (b), that the group $G$ is reduced. If $G$ is a torsion-free group of finte rank, then the fact that $G \in \mathfrak{N}$ (i.e., $G / n G$ is finite for each $n>0$ ) is proved in [1, Theorem 0.1]. The torsion case follows directly from item (e), since a reduced $p$-group of finite rank is finite.

(g) Suppose that $G \in \mathfrak{N}$ is a reduced torsion-free group. Thus $\left(G, v_{G}\right)$ is a Hausdorff totally bounded group. Then its completion $K$ is a compact group. Moreover, for every $n>0$ the closure of the $v_{G}$-open subgroup $n G$ coincides with $n K$ (the density of $n G$ in $n K$ is obvious, so it suffices to note that $n K$ is compact, hence closed, 
as a continuous image of the compact group $K$ under the multiplication by $n$ ). Since $n G$ is closed in $G$, one has $n G=G \cap \overline{n G}=G \cap n K$, this proves that $G$ is a pure subgroup of $K$.

Since, by the definition of completion, the group $K$ has as basic neighbourhoods of 0 the finite-index subgroups $n K=\overline{n G}$, this implies that $\left(K, v_{K}\right)$ is compact. Now Orsatti's theorem [28] applies to provide a topological isomorphism of $K$ with a group of the form $\prod_{p} \mathbb{J}_{p}^{k_{p}}$, where the $k_{p}$ 's are non-negative integers. Conversely, a pure subgroup of a group of this form belongs to $\mathfrak{N}$, by items (a) and (b).

Item (g) of the above theorem shows that if a torsion-free reduced group $G$ belongs to $\mathfrak{N}$, then $|G| \leq 2^{\aleph_{0}}$. On the other hand, all non-trivial groups of the form $G=\prod_{p} \mathbb{J}_{p}^{k_{p}}$ obviously belong to $\mathfrak{N}$ and are torsion-free, reduced and of size $2^{\aleph_{0}}$.

The class $\mathfrak{N}$ of narrow groups is not closed under taking arbitrary subgroups; for instance, $\mathbb{J}_{p} \in \mathfrak{N}$, but a free subgroup of $\mathbb{J}_{p}$ of infinite rank does not belong to $\mathfrak{N}$ (obviously, a free abelian group belongs to $\mathfrak{N}$ if and only if has finite rank). The next corollary determines the hereditary core of $\mathfrak{N}$, namely, the largest subclass of $\mathfrak{N}$ closed under taking arbitrary subgroups.

Corollary 4.4. All subgroups of a group $G$ belong to $\mathfrak{N}$ if and only if $G$ is a group with finite ranks.

Proof. By the definition of rank, it is obvious that the class of groups with finite ranks is closed under taking subgroups, and item (b) in Theorem 4.3 ensures that these groups are narrow. So, assuming that all subgroups of a group $G$ belong to $\mathfrak{N}$, we must prove that $r k_{0}(G)$ and $r k_{p}(G)$ are finite for all primes $p$. The divisible part $d(G)$ is a group with finite ranks, otherwise it contains either a free group of infinite rank, or an elementary $p$-group of infinite dimension, which fail to be narrow. Hence we can assume that $G$ is reduced. By item (d), it is enough to separately check $t(G) \in \mathfrak{N}$ and $G / t(G) \in \mathfrak{N}$. Item (e) gives the conclusion for $t(G)$, while if $G$ is torsion-free it must have finite rank, by the same argument used for divisible groups.

We denote the subclass of $\mathfrak{N}$ consisting of the groups with finite ranks by $\mathfrak{F} \mathfrak{R}$. We have seen that the following inclusion holds:

$$
\mathfrak{F} \mathfrak{R} \cup \mathfrak{O} \subseteq \mathfrak{N} \text {. }
$$

Note that a countable group $G$ belongs to $\mathfrak{O}$ if and only it is finite, since the infinite compact groups have size at least $2{ }^{\aleph_{0}}$. Hence, the above inclusion is strict, witnessed by the group $\bigoplus_{\aleph_{0}} \mathbb{Q}$ (or any other countably infinite divisible group of infinite torsion-free rank). The intersection $\mathfrak{F} \mathfrak{R} \cap \mathfrak{O}$ consists of the finite groups.

\section{Positive answers to Conjecture 1.6}

In this section we prove that Conjecture 1.6 holds for groups with finite ranks and for Orsatti groups, i.e., for groups in the class $\mathfrak{F} \mathfrak{R} \cup \mathfrak{O}$ considered above. We start with a result regarding uniformly fully inert subgroups which are narrow.

Lemma 5.1. Let $H$ be a uniformly fully inert subgroup of the group $G$.

1. if $H \in \mathfrak{N}$, then $H / H \star$ is finite;

2. if furthermore $H^{\star}$ is torsion-free and $H$ has finite rank, then also $H^{\star} / H$ is finite.

In both cases, $H$ is commensurable with a fully invariant subgroup of $G$.

Proof. 1. If $H$ has inertial bound $n$, then the quotient $H / H_{\star}$ is bounded by $n$ by Lemma 2.10. As bounded quotients of narrow groups are finite, $H / H \star$ is finite.

2. By Lemma 2.10, the quotient $H^{\star} / H$ is bounded by $n$, and $n H^{\star}$, as a subgroup of $H$, has also finite rank. Since $H^{\star} \cong n H^{\star}, H^{\star}$ has finite rank, so it is narrow. By the same argument as above, $H^{\star} / H$ is finite. 
Remark 5.2. It is not possible to eliminate the hypothesis that $H^{*}$ is torsion-free in item (2) of Lemma 5.1. This is witnessed by $G=\mathbb{Z} \oplus \bigoplus_{i \geq 0}\left\langle e_{i}\right\rangle$ with $o\left(e_{i}\right)=p$ for all $i$. Indeed, if $H=\mathbb{Z}$, then $H^{\star}=G$ and $H_{\star}=p G$, hence $H^{\star} / H \cong \bigoplus_{i \geq 0}\left\langle e_{i}\right\rangle$ is infinite.

From Lemma 5.1 we immediately derive the following

Corollary 5.3. If $H$ is a uniformly fully inert subgroup of finite rank of a torsion-free group $G$, then both $H / H$ * and $H^{\star} / H$ are finite. Consequently, $H$ is commensurable with a fully invariant subgroup of $G$.

Lemma 5.1 enables us to prove Conjecture 1.6 for arbitrary groups with finite ranks; note that this fact provides an immediate proof of the implication (3) $\rightarrow$ (1) in [8, Theorem 5.13].

Proposition 5.4. If $G \in \mathfrak{F} \Re$, then $\operatorname{Jn} \tilde{v^{\sim}}(G)=\mathcal{J}_{u}(G)=\mathcal{J} n v \star(G)$.

Proof. Let $H$ be a uniformly fully inert subgroup of $G \in \mathfrak{F} \mathfrak{R}$. Then $H \in \mathfrak{F} \mathfrak{R}$ as well, according to Corollary 4.4. Now Lemma 5.1 implies that $H / H_{\star}$ is finite, i.e., $H \in J n v_{\star}(G)$. By Lemma 2.9, this proves that $\mathrm{J} n \tilde{v^{2}}(G)=$ $\mathcal{J}_{u}(G)=\operatorname{Jn} v_{\star}(G)$.

The next example shows the existence of a torsion-free group $G$ of infinite rank with $J_{n} \tilde{v^{\prime}}(G)=\mathcal{J}_{u}(G)$ that is neither free nor divisible.

Example 5.5. For the group $\mathbb{J}_{p}$ of the $p$-adic integers it follows that

$$
\operatorname{Inv}\left(\mathbb{J}_{p}\right)=\operatorname{Jn} \tilde{v}\left(\mathbb{J}_{p}\right)=\mathcal{J}_{u}\left(\mathbb{J}_{p}\right) .
$$

Proof. As fully invariant subgroups of $\mathbb{J}_{p}$ are ideals of the ring $\mathbb{J}_{p}$, it follows that $J_{n} v\left(\mathbb{J}_{p}\right)=\{0\} \cup\left\{p^{n} \mathbb{J}_{p} \mid n \geq 0\right\}$. Let now $H$ be a non-zero uniformly fully inert subgroup of $\mathbb{J}_{p}$. Since $H^{*} / H$ is bounded, by Lemma 2.10, and $H^{\star}=p^{k} \mathbb{J}_{p}$ for some $k \geq 0$, it follows that $p^{n} \mathbb{J}_{p} \leq H$ for some $n \geq 0$. But the only subgroups between $p^{k} \mathbb{J}_{p}$ and $p^{n} \mathbb{J}_{p}$ are of the form $p^{m} \mathbb{J}_{p}$ for some $k \leq m \leq n$, as $\mathbb{J}_{p} / p^{n} \mathbb{J}_{p}$ is a finite $p$-group. So we have proved that $\operatorname{Jn} v\left(\mathbb{J}_{p}\right)=\mathcal{J}_{u}\left(\mathbb{J}_{p}\right)$.

For a discussion on the issue of whether one can add to the chain of equalities (5.1) also $\mathcal{J}\left(\mathbb{J}_{p}\right)$, see Questions 7.8 and 7.9 .

The preceding Example 5.5 is the starting point to prove the following results concerning local Orsatti groups. By a local Orsatti group we mean an Orsatti group which is a (finitely generated) $\mathbb{J}_{p}$-module for some $p$.

Proposition 5.6. If $G$ is a local Orsatti group, then every uniformly fully inert subgroup is either finite or of finite index, therefore $\operatorname{Jn} \tilde{v^{\prime}}(G)=\mathcal{J}_{u}(G)$.

Proof. First we prove the claim in the torsion-free case. In [24] it is proved that a non-zero submodule $H$ of a free $\mathbb{J}_{p}$-module $A$ of finite rank $r$ belongs to $\mathcal{J}(G)$ if and only if $H$ has finite index in $A$. Here we must assume that $H$ is just a non-zero subgroup, and not a submodule, of the free $\mathbb{J}_{p}$-module $A$, but we have the stronger assumption that $H$ is uniformly fully inert; we want to prove that still $H$ has finite index in $A$. The case $r=1$ is covered by Example 5.5. Let $A=\bigoplus_{1 \leq i \leq r} A_{i}$, where $r>1$ and $A_{i} \cong \mathbb{J}_{p}$ for all $i$. Let $\pi_{i}: A \rightarrow A_{i} \leq A$ be the canonical projections.

Now we prove that $H \cap A_{i} \neq 0$ for all $i$. This is ensured, by Corollary 2.7(b) applied to $\psi=\pi_{i}$, for all $i$ with $\pi_{i}(H) \neq 0$ (as $\pi_{i}(H) \neq 0$ implies that $\pi_{i}(H)$ is infinite, since $A_{i} \cong \mathbb{J}_{p}$ is torsion-free). Since $H \neq\{0\}$, there exists an index $j$ such that $\pi_{j}(H) \neq 0$. For every index $i$ fix an isomorphism $\phi_{i}: A_{j} \rightarrow A_{i}$; then $\psi_{i}=\phi_{i} \cdot \pi_{j}$ is a homomorphism $A \rightarrow A_{i}$ such that $\pi_{i}(H) \neq 0$, so $\pi_{i}(H)$ is infinite, hence Lemma 2.7(b) can be applied for $\psi=\psi_{i}$ to entail $H \cap A_{i} \neq 0$.

From Corollary 2.7(a) and Example 5.5 we derive that $H \cap A_{i}=p^{n_{i}} A_{i}$, with $n_{i}>0$ for all $i \leq r$, so $H \geq$ $\bigoplus_{1 \leq i \leq r} p^{n_{i}} A_{i}$. The last group is of finite index in $A$, so such is $H$. 
Let now $G=A \oplus F$ be a local Orsatti group, where $A$ is as above and $F$ is a finite $p$-group, and let $H$ be a non-zero uniformly fully inert subgroup of $G$. If $H \cap A=0$, then $H$ embeds in $F$, so it is finite. Otherwise, $H \cap A \neq 0$ has finite index in $A$, by Corollary 2.7 (a) and what proved above, therefore $G / H$, as an epic image of $(A / A \cap H) \oplus(F / F \cap H)$, is finite. Thus we conclude that $H$ is either finite, or of finite index in $G$, as desired, hence $\operatorname{Jn} \tilde{v^{\sim}}(G)=\mathcal{J}_{u}(G)$.

We are now in the position to prove the main result of this section.

Theorem 5.7. A uniformly fully inert subgroup H of an Orsatti group $G=\prod_{p} G_{p}$, where $G_{p}$ is a finitely generated $\mathbb{J}_{p}$-module, is commensurable with a fully invariant subgroup of $G$.

Proof. Let $n$ be the inertial bound of $H$ and $\mathbb{P}_{1}=\{p \in \mathbb{P}: p \mid n\}, \mathbb{P}_{2}=\{p \in \mathbb{P}: p \nvdash n\}$. Let us set

$$
G_{1}=\prod_{p \in \mathbb{P}_{1}} G_{p} \text { and } G_{2}=\prod_{p \in \mathbb{P}_{2}} G_{p} .
$$

Then $G=G_{1} \oplus G_{2}$ and each $G_{i}$ is a fully invariant subgroup of $G$. Since $n$ is invertible modulo $p \in \mathbb{P}_{2}$, we can apply Proposition 2.8 and derive, using its notation, that $H=H_{1} \oplus H_{2}$ with $H_{2}$ a fully invariant subgroup of $G_{2}$. In order to conclude that $H \in J n \tilde{v}(G)$, according to item $\left(2_{c}\right)$ in Proposition 2.8, it is sufficient to check that $H_{1} \in \operatorname{Jnv} \tilde{\sim}\left(G_{1}\right)$.

For every $p \in \mathbb{P}_{1}$, let $\pi_{p}: G \rightarrow G_{p}$ be the projection. Applying again Proposition 2.8 to the subgroup $H_{1}$ of $G_{1}$, we deduce that each $\pi_{p}\left(H_{1}\right)$ is a uniformly fully inert subgroup of $G_{p}$ for all $p \in \mathbb{P}_{1}$. By Proposition 5.6, we know that $\pi_{p}(H)$ is either finite, or of finite index in $G_{p}$. The hypothesis that $H$ is uniformly fully inert in $G$, by using the projections $\pi_{p}: G \rightarrow G_{p}\left(p \in \mathbb{P}_{1}\right)$, yields:

$$
\bigoplus_{p \in \mathbb{P}_{1}} n \pi_{p}\left(H_{1}\right) \leq H_{1} \leq \bigoplus_{p \in \mathbb{P}_{1}} \pi_{p}\left(H_{1}\right)
$$

Being $\mathbb{P}_{1}$ a finite set, we obtain that $H_{1} \in \operatorname{Jn} \tilde{v^{\sim}}\left(G_{1}\right)$.

\section{When $\mathcal{J}_{u}(G) \subsetneq \mathcal{J}(G)$ : fully inert but not uniformly fully inert subgroups}

The goal of this section is to prove that the examples of $p$-group $G$ and torsion-free $\rrbracket_{p}$-module $X$, exhibited in [24] and [25] respectively, which demonstrate the strict inclusions

$$
\operatorname{Jn} \tilde{v^{\prime}}(G) \subsetneq \mathcal{J}(G) \text { and } \operatorname{Jn} \tilde{v^{2}}(X) \subsetneq \mathcal{J}(X)
$$

also provide the proof of the sharper strict inclusions

$$
\mathcal{J}_{u} \tilde{u}(G) \subsetneq \mathcal{J}(G) \text { and } \mathcal{J}_{u} \tilde{u}(X) \subsetneq \mathcal{J}(X) .
$$

Hence these two examples do not disprove our Conjecture 1.6.

Recall that a basic subgroup $B$ of a $p$-group $G$ is called semi-standard if $B=\bigoplus_{n \geq 1} B_{n}$, with $B_{n}$ either zero or isomorphic to the direct sum of $f_{n}$ copies of $Z\left(p^{n}\right)$ for some positive integers $f_{n}$. The integers $f_{n}$ coincide with the so-called Ulm-Kaplansky invariants (of finite index) of $B$, and the direct summands $B_{n}$ are called the homogeneous components of $B$.

In [24, Theorem 4.2] it is proved that, given a reduced separable $p$-group $G$ of cardinality $2^{\aleph_{0}}$ with semistandard basic subgroup $B$, and with endomorphism $\operatorname{ring} \operatorname{End}(G)=\mathbb{J}_{p} \cdot 1_{G} \oplus E_{s}(G)$, where $E_{s}(G)$ is the twosided ideal of the small endomorphisms (see [21, Chapter 7, Section 3] for its definition), the socle $B[p]$ of the basic subgroup $B$ is infinite and fully inert, but it fails to be commensurable with a fully invariant subgroup of $G$. The crucial point in the proof is that $B$ is countable and any fully invariant non-trivial subgroup of $G$ has cardinality $2^{\aleph_{0}}$. 
Thus the proper inclusion $\operatorname{In} \tilde{v}(G) \subsetneq \mathcal{J}(G)$ is proved for such a group $G$, and it is natural to ask whether the subgroup $B[p]$ belongs to $J_{u}(G)$, i.e., whether it is uniformly fully inert. In order to prove that this fails to be true, we need the following lemma.

Lemma 6.1. Let $G$ be an unbounded p-group with a basic subgroup $B$ such that $r k(G / B) \geq r k(B)$, and let $B=\bigoplus_{m} B_{m}$ be the direct decomposition of $B$ into homogeneous components. Then, for every integer $n \geq 1$, there exists an endomorphism $\phi$ of $G$ such that its restriction to $B_{1} \oplus \cdots \oplus B_{n}$ is injective, and $\phi\left(B_{1} \oplus \cdots \oplus B_{n}\right) \cap B=0$.

Proof. Since $G / B \cong \bigoplus_{\alpha} \mathbb{Z}\left(p^{\infty}\right)$ and $r k(G / B) \geq r k(B)$, there exists a subgroup $H / B$ of $G / B$ such that $H / B \cong B$. Let

$$
B=\bigoplus_{i \in I}\left\langle b_{i}\right\rangle \text { and } H / B=\bigoplus_{i \in I}\left\langle h_{i}+B\right\rangle,
$$

with $\left\langle b_{i}\right\rangle \cong\left\langle h_{i}+B\right\rangle$ for all $i \in I$. If the common order of $b_{i}$ and $h_{i}+B$ is $p^{m_{i}}\left(m_{i} \geq 1\right)$, then $p^{m_{i}} h_{i} \in B \cap p^{m_{i}} G=$ $p^{m_{i}} B$, by the purity of $B$ in $G$; hence there exist elements $y_{i} \in B$ such that $p^{m_{i}}\left(h_{i}-y_{i}\right)=0$ for all $i \in I$. Set $x_{i}=h_{i}-y_{i}$; then

$$
o\left(x_{i}\right)=p^{m_{i}} .
$$

Indeed, obviously, $p^{m_{i}} x_{i}=0$. If $p^{d} x_{i}=0$ for some $0 \leq d<m_{i}$, then $p^{d} h_{i}=p^{d} y_{i} \in B$ implies $d=0$, as $o\left(h_{i}+B\right)=p^{m_{i}}$ in $H / B$. This proves (6.1).

With $L=\left\langle x_{i}: i \in I\right\rangle=\sum_{i}\left\langle x_{i}\right\rangle$ we claim that

$$
B \cap L=\{0\}
$$

and the elements $x_{i}(i \in I)$ are independent, i.e.,

$$
L=\bigoplus_{i \in I}\left\langle x_{i}\right\rangle
$$

Indeed, if $\sum_{i \in F} a_{i} x_{i} \in B$, where $F$ is a finite subset of $I$ and $a_{i} \in \mathbb{Z}$ for all $i \in F$, then

$$
\sum_{i \in F} a_{i} h_{i}=\sum_{i \in F} a_{i} y_{i} \in B
$$

implies that $\sum_{i \in F} a_{i}\left(h_{i}+B\right)=0$, hence $p^{m_{i}}$ divides $a_{i}$ for all $i \in F$, therefore $a_{i} x_{i}=0$, in view of (6.1). This proves both (6.2) and (6.3).

Note that (6.3) obviously provides an isomorphism

$$
\eta: B \rightarrow L
$$

by sending the generators $b_{i}$ of $B$ to the corresponding generators $x_{i}$ of $L$. Moreover, $\eta(B)=L$ trivially meets $B$ in view of (6.2).

To conclude, extend the restriction

$$
\xi:=\eta \uparrow_{B_{1} \oplus \cdots \oplus B_{n}}: B_{1} \oplus \cdots \oplus B_{n} \rightarrow G
$$

of the isomorphism (6.5) to an endomorphism $\phi$ of $G$ making use of the well-known fact that $B_{1} \oplus \cdots \oplus B_{n}$ is a direct summand of $G$, so $\xi$ can be extended by sending to 0 a complement of $B_{1} \oplus \cdots \oplus B_{n}$. Then $\phi$ is the desired endomorphism, as $\phi\left\lceil_{B_{1} \oplus \cdots \oplus B_{n}}=\xi\right.$ is injective and $\phi(B) \leq L$ trivially meets $B$.

We can now prove that in Theorem 4.2 in [24] the socle $B[p]$ of the basic subgroup $B$ is not uniformly fully inert.

Proposition 6.2. Let $G$ be an uncountable reduced separable p-group $G$ with semi-standard basic subgroup $B$, and with endomorphism ring $\operatorname{End}(G)=\mathbb{J}_{p} \cdot 1_{G} \oplus E_{s}(G)$. Then the socle $B[p]$ of $B$ is fully inert in $G$, but it is not uniformly fully inert. 
Proof. The subgroup $B[p]$ is fully inert, by [24, Theorem 4.2].

To prove that $B[p] \notin \mathcal{J}_{u}(G)$ we must show that for every $k \in \mathbb{N}$ there exists an endomorphism $\phi$ of $G$, depending on $k$, such that $|(B[p]+\phi(B[p])) / B[p]| \geq k$. Choose a positive integer $N$ such that $\left|\left(B_{1} \oplus \cdots \oplus B_{n}\right)[p]\right| \geq$ $k$. Since $B$ is countable, one has

$$
|G|=|G / B|=r k(G / B)>|B| \geq r k(B) .
$$

Therefore, Lemma 6.1 apples to produce an endomorphism $\phi$ of $G$ such that its restriction to $B_{1} \oplus \cdots \oplus B_{n}$ is injective, and $\phi\left(B_{1} \oplus \cdots \oplus B_{n}\right) \cap B=0$. Then $\phi$ satisfies the desired inequality $|(B[p]+\phi(B[p])) / B[p]| \geq k$, since $\phi\left(B_{1} \oplus \cdots \oplus B_{n}\right)[p] \cong\left(B_{1} \oplus \cdots \oplus B_{n}\right)[p]$ and $\phi\left(B_{1} \oplus \cdots \oplus B_{n}\right)[p] \cap B[p]=0$.

The $p$-groups satisfying the requirements of Proposition 6.2 are the so-called Pierce-like groups; they were first constructed in [29, Theorem 15.4]. The natural problem arises: does the equality $\operatorname{Jn} \tilde{v^{2}}(G)=\mathcal{J}_{u}(G)$ hold true for the $p$-group $G$ in Proposition 6.2?

We pass now to torsion-free $\mathbb{I}_{p}$-modules. It is well known that the non-zero fully invariant submodules of a torsion-free reduced $\mathbb{J}_{p}$-module $M$ are the obvious ones, that is, those of the form $p^{k} M$ for some non-negative $k$ (see [26, Exercise 72(b)]).

In [25] it is proved that there exists a torsion-free $\mathbb{J}_{p}$-module $X$ containing a fully inert submodule $H$ which is non-commensurable with $p^{k} X$, for all non-negative $k$. The $\mathbb{J}_{p}$-module $X$, furnished by a realization theorem of commutative rings as endomorphism rings of $\mathbb{J}_{p}$-modules (see [22, Theorem 4.1]), has its endomorphism ring satisfying $\operatorname{End}(X)=\mathbb{J}_{p} \cdot 1_{X} \oplus E_{0}(X)$, where $E_{0}(X)$ is the two-sided ideal of the endomorphisms with finite rank image. The required submodule $H$ is any $H \leq X$ such that $p X<H$ and both $X / H$ and $H / p X$ are infinite. Thus the proper inclusion $\mathfrak{J} n \tilde{v}(X) \subsetneq \mathcal{J}(X)$ is proved for such a $\mathbb{J}_{p}$-module $X$.

We prove that the submodule $H$ does not belong to $\mathcal{J}_{u}(X)$.

Proposition 6.3. Let $X$ be a reduced torsion-free $\mathbb{J}_{p}$-module with endomorphism ring $\operatorname{End}(X)=\mathbb{J}_{p} \cdot 1_{X} \oplus E_{0}(X)$. If a submodule $H$ of $X$ satisfies $p X<H<X$ and both $X / H, H / p X$ are infinite, then $H$ is is not uniformly fully inert.

Proof. In order to prove that $H \notin \mathcal{J}_{u}(X)$, it is enough to find for every $k \in \mathbb{N}$ an endomorphism $\phi_{k}$ of $X$, such that

$$
\left|\left(H+\phi_{k} H\right) / H\right| \geq k .
$$

Fix a $k \in N$ and let $\pi: X \rightarrow X / p X$ be the canonical homomorphism. The subspace $\pi(H)=H / p X$ of the vector space $X / p X$ splits, let $X / p X=H / p X \oplus K / p X$, where $K$ is a $\mathbb{J}_{p}$-submodule of $X$ containing $p X$. Our hypotheses on $H$ imply that both $H / p X$ and $K / p X$ are infinite-dimensional. Let

$$
\mathcal{H}=\left\{h_{i}: i \in \mathbb{N}\right\} \text { and } \mathcal{K}=\left\{k_{i}: i \in \mathbb{N}\right\}
$$

be two sequences in $H$ and $K$, respectively, such that the sets $\left\{\pi\left(h_{i}\right)\right\}_{i \in \mathbb{N}}$ and $\left\{\pi\left(k_{j}\right)\right\}_{j \in \mathbb{N}}$ are linearly independent in the vector spaces $H / p X$ and $K / p X$.

Choose $n \in \mathbb{N}$ with $p^{n+1} \geq k$ and let $H_{0}$ be the $\mathbb{J}_{p}$-submodule of $X$ generated by $h_{0}, h_{1}, \ldots h_{n}$. By the choice of $\mathcal{H}$, the submodule $H_{0}$ is pure and free, so $H_{0}=h_{0} \mathbb{J}_{p} \oplus \cdots \oplus h_{n} \mathbb{J}_{p}$. As $\mathbb{J}_{p}$, hence $H_{0}$ as well, is pure-injective, this yields that $H_{0}$ is a direct summand of $X$. Therefore, the map sending $h_{0}, h_{1}, \ldots, h_{n}$, respectively, to the elements $k_{0}, k_{1}, \ldots, k_{n}$ of $K$ can be extended to an endomorphism $\phi_{k}$ of $X$ sending the complement of $H_{0}$ to zero. By the choice of $\mathcal{K}$,

$$
\left(H+\phi_{k} H\right) / H \cong\left(\left(H+\phi_{k} H\right) / p X\right) /(H / p X)
$$

has cardinality $p^{n+1} \geq k$. This proves that $\phi_{k}$ satisfies (6.6).

The next consequence of Proposition 6.3 shows that also for the module $X$, which provides an example for the strict inequality $\mathcal{J} n \tilde{v}(X) \subsetneq \mathcal{J}(X)$, the equality $\mathfrak{J} n \tilde{v}(X)=\mathcal{J}_{u}(X)$ of Conjecture 1.6 holds true. 
Theorem 6.4. Let $X$ be the $\mathbb{J}_{p}$-module of Proposition 6.3. Then any non-zero uniformly fully inert submodule $K$ of $X$ is commensurable with a submodule of the form $p^{k} X$ for some non-negative $k$. Therefore $\operatorname{Jn}_{\tilde{v}}(X)=\mathcal{J}_{u}(X)$.

Proof. Let us note that we can replace $K$, if necessary, by a subgroup of $K$ which is commensurable with $K$.

Since $K$ is uniformly fully inert the quotient $K^{\star} / K$ is bounded, hence, for a suitable $m>0$,

$$
p^{m} K^{\star} \leq K_{\star}<K<K^{\star} .
$$

In [25] it is proved that every non-zero fully invariant submodule of $X$ is of the form $p^{k} X$ for some $k \geq 0$, hence there exist a minimal positive integer $s$ and a maximal $r>s$ such that

$$
p^{s} X<K<p^{r} X .
$$

Let $d:=r-s$. If $d=1$, then we intend to apply Proposition 6.3 to deduce that $K \in \mathcal{J} n \tilde{v^{\sim}}(X)$. To this end we note that $K$ is a uniformly fully inert submodule of $p^{r} X$ as well since every endomorphism of $p^{r} X$ (uniquely) extends to an endomorphism of $X$. Hence, Proposition 6.3 applied to $p^{r+1} X<K<p^{r} X$ implies that either $p^{r} X / K$ or $K / p^{r+1} X$ are finite, hence $K \in J n \tilde{v^{2}}\left(p^{r} X\right)$. As $p^{r} X$ is fully invariant in $X$, this yields $K \in \mathcal{J} n \tilde{v}(X)$ as well.

Now assume $d>1$ and the assertion true for all submodules $K$ of $X$ with

$$
p^{v} X<K<p^{u} X \text { for some naturals } u<v \text { with } v-u<d .
$$

Using the fact that we can replace $K$ by a subgroup of it which is commensurable with $K$, we can assume, without loss of generality, that $\left(K+p^{r+1} X\right) / p^{r+1} X$ is infinite. Indeed, let $K_{1}:=K \cap p^{r+1} X$ and assume that $\left(K+p^{r+1} X\right) / p^{r+1} X \cong K / K_{1}$ is finite. Then $K_{1} \sim K$ and $p^{s} X<K_{1}<p^{r+1} X$. As $s-(r+1)<d, K_{1} \in J_{n v} \sim(X)$, by our inductive hypothesis. Hence, $K \in \operatorname{In} \tilde{v}(X)$ as well.

From now on we assume that $\left(K+p^{r+1} X\right) / p^{r+1} X$ is infinite.

Let us see that if $p^{r} X /\left(K+p^{r+1} X\right)$ is finite, then $\left.K \in \mathcal{J} n v \tilde{(} X\right)$. Indeed, in such a case $p^{r} X=p^{r+1} X+K+F$ for some finitely generated subgroup $F$ of $X$. Multiplying $s-r-1$ times by $p$ and replacing one gets $p^{r} X=$ $p^{s} X+K+F=K+F$, as $K \geq p^{s} X$. Since $F$ is finitely generated, we deduce that $p^{r} X / K$, being isomorphic (as an abelian group) to a quotient of $F$, is countable. As $p^{r} X / K$ is a finitely generated $\mathbb{J}_{p}$-module and $\left|\mathbb{J}_{p}\right|=\mathfrak{c}$, this yields that $p^{r} X / K$ is finite. This implies that $K \in \operatorname{Jn} v^{\sim}(X)$.

From now on we assume that $p^{r} X /\left(K+p^{r+1} X\right)$ is infinite and we shall see that this leads to a contradiction with our assumption that $K$ is uniformly fully inert. Our next step is adapting the proof of Proposition 6.3 to obtain, for each positive integer $n$, an endomorphism $\phi$ of $X$ such that $|(K+\phi K) / K| \geq n$. To this end choose elements $p^{r} x_{1}, \ldots, p^{r} x_{n} \in K$ which are independent modulo $p^{r+1} K$, using the fact that $\left(K+p^{r+1} X\right) / p^{r+1} X$ is infinite. The elements $x_{1}, \ldots, x_{n}$ generate a direct summand $H$ of $X$. Now use the fact that $p^{r} X /\left(K+p^{r+1} X\right)$ is infinite, so there exist independent elements

$$
p^{r} y_{1}+p^{r+1} X, \ldots, p^{r} y_{n}+p^{r+1} X \in p^{r} X / p^{r+1} X
$$

in the complement of $\left(K+p^{r+1} X\right) / p^{r+1} X$. The assignment which sends each element $x_{i}$ to $y_{i}$, for $1 \leq i \leq n$, can be extended to an endomorphism $\phi$ of $X$ which sends the complement of $H$ to zero; then the desired inequality $|(K+\phi K) / K| \geq n$ holds.

\section{Conclusion}

In this section we summarize the results concerning Conjecture 1.6 on uniformly fully inert subgroups obtained up to now, and we list a series of open questions.

\subsection{The state of art}

For several classes of groups $G$ the equality $\operatorname{In} \tilde{v^{2}}(G)=\mathcal{J}(G)$ holds, hence, a fortiori, also the equality $\operatorname{Inv}(G)=$ $\mathcal{J}_{u}(G)$ of Conjecture 1.6 holds. The list of these classes includes: 
- free groups (see [19]);

- direct sums of cyclic $p$-groups (see [24]);

- divisible groups $D$ such that $r_{0}(D)$ is either zero or $\infty$ (see Theorem 3.2);

- completely decomposable groups of finite rank $G$ such that their rank one summands have types which are either equal or incomparable and contain no $\infty$ 's (see Theorem 3.4).

Furthermore, for divisible groups and completely decomposable groups of finite rank not satisfying the conditions above, we know that the equality of Conjecture 1.6 still holds.


do not furnish counter-example to Conjecture 1.6 (see Section 6).

Finally, there are groups $G$ for which we only know that the equality of Conjecture 1.6 holds. These groups are:

- the torsion-free groups of finite rank (see Proposition 5.4);

- the Orsatti groups (see Theorem 5.6).

\subsection{Open questions}

For further investigation of Conjecture 1.6 we propose the following questions.

The first series of questions concerns Theorem 5.7 asserting that an Orsatti group $G$ satisfies our main conjecture, i.e., every uniformly fully inert subgroup of $G$ is commensurable with a fully invariant subgroup.

Question 7.1. Can Theorem 5.7 be extended to the groups of the form $G=\mathbb{J}_{p}^{\mathbb{N}}$ ? What about arbitrary infinite powers $\mathbb{J}_{p}^{K}$ ?

Question 7.2. Can Theorem 5.7 be extended to splitting $\mathbb{J}_{p}$-modules?

Question 7.3. Can we extend Theorem 5.7 from Orsatti groups to arbitrary algebraically compact groups, or, more generally, to cotorsion groups, which have a decomposition as direct products of p-primary components?

The next question concerns the class of completely decomposable groups. A positive answer was conjectured after Theorem 3.6.

Question 7.4. Are the equivalent conditions of Theorem 3.6 equivalent also to the equality $\mathcal{J}(G)=\mathcal{J}_{u}(G)$, i.e., every fully inert subgroup is uniformly fully inert?

The following question was posed after Proposition 6.2.

Question 7.5. Does the equality $\mathrm{J} \tilde{v^{\sim}}(G)=\mathcal{J}_{u}(G)$ hold true for the p-group $G$ in Proposition 6.2?

It is probably hopeless to prove Conjecture 1.6 for all abelian groups. A more reasonable strategy is to test various reasonably chosen classes of groups.

Question 7.6. Is Conjecture 1.6 true for

(a) all completely decomposable abelian groups?

(b) all torsion-free abelian groups?

(c) all torsion abelian groups?

(d) all splitting abelian groups?

(e) all narrow groups?

To answer (c) it suffices to consider the problem for all $p$-groups, when $p$ ranges over all primes. 
Question 7.7. Is the analogue of Conjecture 1.6 true for $\mathbb{J}_{p}$-modules?

Our next question is related to the equality $\mathfrak{I n v}\left(\mathbb{J}_{p}\right)=\mathfrak{J} n \tilde{v}\left(\mathbb{J}_{p}\right)=\mathcal{J}_{u}\left(\mathbb{J}_{p}\right)$ established in Example 5.5.

Question 7.8. Determine the fully inert subgroups of $\mathbb{J}_{p}$. In particular, is $\mathcal{J}\left(\mathbb{J}_{p}\right)$ equal to $J_{n} v\left(\mathbb{J}_{p}\right)$, or do there exist fully inert non-trivial subgroups $H$ of $\mathbb{J}_{p}$ distinct from $p^{k} \mathbb{J}_{p}$ for any $k$ ?

Note that if $H$ is a fully inert non-trivial subgroup of $\mathbb{J}_{p}$, then $\mathbb{J}_{p} / H$ is torsion, since $H^{\star} / H$ is torsion and $H^{\star}=$ $p^{k} \mathbb{J}_{p}$ for some $k$. This motivates the question of whether the converse is true.

Question 7.9. Is every subgroup $H$ of $\mathbb{J}_{p}$ such that $\mathbb{J}_{p} / H$ is torsion necessarily fully inert?

Acknowledgement: Dikran Dikranjan thankfully acknowledges financial support of the National Group for Algebraic and Geometric Structures, and their Applications (GNSAGA - INdAM).

\section{References}

[1] D. Arnold, Finite Rank Torsion Free Abelian Groups and Rings, Lecture Notes in Mathematics, 931. Springer-Verlag, BerlinNew York, 1982.

[2] V. V. Belyaev, Inert subgroups in simple locally finite groups, in "Finite and locally finite groups" (Istanbul, 1994), pp. 213218, Kluwer Acad. Publ., Dordrecht, 1995.

[3] G. M. Bergman, H. W. Lenstra Jr., Subgroups close to normal subgroups, J. Algebra, 127 (1989), no. 1, 80-97.

[4] C. Casolo, U. Dardano, S. Rinauro, Groups in which each subgroup is commensurable with a normal subgroup, J. Algebra 496 (2018), 48-60.

[5] I. Castellano, G. Corob Cook, and P.H. Kropholler A property of the lamplighter group, to appear on Topological Algebra and its Applications, this issue.

[6] A.R. Chekhlov, Fully inert subgroups of completely decomposable finite rank groups and their commensurability, Vestn. Tomsk. Gos. Univ. Mat. Mekh. 41 (2016), no. 3, $42-50$ (in Russian).

[7] A. R. Chekhlov, On Fully Inert Subgroups of Completely Decomposable Groups, Math. Notes. 101 (2017), 365-373.

[8] U. Dardano, D. Dikranjan, S. Rinauro, Inertial properties in groups, International J. Group Theory, 7 (2018), no. 3, 17-62.

[9] U. Dardano, S. Rinauro, Inertial automorphisms of an abelian group, Rend. Sem. Mat. Univ. Padova, 127 (2012), $213-233$.

[10] U. Dardano, S. Rinauro, Inertial endomorphisms of an abelian group, Ann. Mat. Pura Appl., 195 (2016), no. 1, 219-234.

[11] U. Dardano, S. Rinauro, On the ring of inertial endomorphisms of an abelian group, Ricerche Mat., 63 (2014), no. 1, $103-115$.

[12] D. Dikranjan, Compactness and connectedness in topological groups, Topology Appl., 84 (1998) 227-252.

[13] D. Dikranjan, A. Giordano Bruno, Entropy on abelian groups, Adv. Math. 298 (2016), 612-653.

[14] D. Dikranjan, A. Giordano Bruno, Functorial topologies and finite-index subgroups of abelian groups, Topology Appl. 158 (2011) 2391-2407.

[15] D. Dikranjan, A. Giordano Bruno, L. Salce, Adjoint algebraic entropy, J. Algebra 324 (2010) 442-463.

[16] D. Dikranjan, A. Giordano Bruno, L. Salce, S. Virili, Fully inert subgroups of divisible Abelian groups, J. Group Theory, 16 (2013), 915-939.

[17] D.Dikranjan, A. Giordano Bruno, L. Salce, S. Virili, Intrinsic algebraic entropy, J. Pure Appl. Algebra 219 (2015), no. 7, 29332961.

[18] D. Dikranjan, B. Goldsmith, L. Salce, P. Zanardo, Algebraic entropy of endomorphisms of abelian groups, Trans. Amer. Math. Soc., 361 (2009), 3401-3434.

[19] D. Dikranjan, L. Salce, P. Zanardo, Fully inert subgroups of free Abelian groups, Periodica Math. Hung. 69 (2014), no.1, 69-78.

[20] D. Dikranjan, M. Sanchis, Dimension and entropy in compact topological group, submitted.

[21] L. Fuchs, Abelian Groups, Springer Monographs in Math., 2015.

[22] B. Goldsmith, Essentially indecomposable modules over a complete discrete valuation ring, Rend. Sem. Mat. Univ. Padova 70 (1983), 21-29.

[23] B. Goldsmith, L. Salce, Algebraic entropies for Abelian groups with applications to their endomorphism rings: a survey, in "Groups, Modules, and Model Theory-Surveys and Recent Developments", pp. 135-175, Springer, 2017.

[24] B. Goldsmith, L. Salce, P. Zanardo, Fully inert subgroups of Abelian p-groups, J. Algebra 419 (2014), 332-349.

[25] B. Goldsmith, L. Salce, P. Zanardo, Fully inert submodules of torsion-free modules over the ring of p-adic integers, Colloquium Math. 136 (2014) no.2, 169-178.

[26] I. Kaplansky, Infinite Abelian Groups, Univ. Michigan Press, Ann Arbor, 1954. 
[27] A. Mader, Basic concepts of functorial topologies, in "Abelian group theory" (Oberwolfach, 1981), Lecture Notes in Mathematics 874, pp. 251-271, Springer, Berlin-New York, 1981.

[28] A. Orsatti, Una caratterizzazione dei gruppi abelian compatti o localmente compatti nella topologia naturale, Rend. Sem. Mat. Univ. Padova 39 (1967), 219-225.

[29] R. S. Pierce, Homomorphisms of primary Abelian groups, in "Topics in Abelian Groups", Scott Foresman (1963), 215-310. 